\title{
Simulation of Near Edge X-ray Absorption Fine Structure (NEXAFS) Measurements of CO on Supported Pd Nanoparticles
}

\author{
Chi M. Yim ${ }^{1}$ - Chi L. Pang ${ }^{1}$ Geoff Thornton ${ }^{1}$
}

Published online: 24 March 2016

(c) The Author(s) 2016. This article is published with open access at Springerlink.com

\begin{abstract}
Near edge X-ray absorption fine structure (NEXAFS) measurements of $\mathrm{CO}$ on Pd nanoparticles have been simulated. This was achieved by calculating the $\mathrm{CO}$ $\pi^{*}$ resonance signal of $\mathrm{CO}$ on a nanoparticle both as a function of the angle of incidence $(I$ vs $\theta)$ and the direction of the electric field vector $\mathbf{E}$ of the incident photon beam $(I$ vs $\beta$ ), with the nanoparticle defined as a (111) top facet with $\{111\}$ and $\{100\}$ side facets. The dependence of the $\pi^{*}$ resonance intensity signal of CO covered nanoparticles on the particle geometry and orientation as well as the bond orientation of $\mathrm{CO}$ is examined. In addition, we compare our simulations to a set of C K-edge NEXAFS experimental data obtained from a single Pd nanoparticle decorated with CO. Our simulation predicts that the nanoparticle has a high lateral aspect ratio of $37.7 \pm 4.1$.
\end{abstract}

Keywords Carbon monoxide - Nanoparticle ·

Adsorption · NEXAFS · Simulation

\section{Introduction}

The study of metal nanoparticles supported on oxide surfaces has become a popular topic of research in recent years. This is motivated in part by their applications in heterogeneous catalysts. In the study of model systems

Geoff Thornton

g.thornton@ucl.ac.uk

1 Department of Chemistry and London Centre for Nanotechnology, University College London, 20 Gordon Street, London WC1H 0AJ, UK there are a variety of surface science techniques available that can unravel different aspects of the nanoparticle properties. For instance, one can employ scanning probes (scanning tunneling microscopy (STM) [1], atomic force microscopy [2]), electron microscopy (X-ray photoemission electron microscopy [3], transmission electron microscopy [4], diffraction (photoelectron diffraction [1]) and scattering techniques (grazing-incidence small angle $\mathrm{X}$-ray scattering $[5,6])$ to monitor the morphology of the nanoparticles. To probe molecular adsorbates on nanoparticles, one can use STM to monitor their adsorption sites [7], infrared absorption spectroscopy to investigate their vibrational modes at different sites [8], and microcalorimetry to measure their sticking coefficients as well as adsorption energies on the nanoparticles [9]. To determine molecular orientations on nanoparticles, near-edge X-ray absorption fine structure (NEXAFS) is one of the few experimental tools available.

Developed in conjunction with the rapid advancement of synchrotron radiation techniques in 1980s, NEXAFS has proven a valuable tool in the determination of molecular orientations on surfaces [10]. The technique relies on dipole selection rules, associated with a photoelectron originating from an $s$ initial state (K edge) being excited into a final-state, $p$-like orbital. For a diatomic molecule, the final-state (anti-bonding) orbital can be of either $\pi^{*}$ or $\sigma^{*}$ type. If $\delta$ is the angle between the electric field vector $\mathbf{E}$ and the direction of the final state orbital $\mathbf{O}$, i.e., the direction of maximum orbital amplitude, the transition intensity $I$ varies as $\cos ^{2} \delta$ [11]. Also, as the direction of a $\pi^{*}$ anti-bonding orbital relative to the corresponding intramolecular bond axis is different from that of a $\sigma^{*}$ orbital, the $\pi^{*}$ resonance exhibits a polarization dependence which is opposite to that of the $\sigma^{*}$ resonance. Such opposition in polarization dependence has been illustrated in previous 
NEXAFS studies, for instance from $\mathrm{CO} / \mathrm{Cu}(110)$ [12] and $\mathrm{CO} / \mathrm{NiO}(100)$ [13].

NEXAFS has been used extensively to study the bonding orientations of molecules that are physisorbed or chemisorbed on many different surfaces, including those of metals [14, 15], oxides [13, 16-18], and semiconductors $[19,20]$. However, it is rarely applied to the study of adsorbates on supported nanoparticles. The reasons are twofold. First, the nanoparticles usually have a variety of geometries and orientations relative to the incident photon beam. Hence, molecules on different nanoparticles can provide varying contributions to the total X-ray absorption signal integrated over the whole sample. Secondly, even in an ideal system that consists only of a single nanoparticle, the polarization dependence of the X-ray absorption signal from different parts (facets, edges and corners) of the nanoparticle can vary significantly from one another. Both of the above factors make it difficult to determine the molecular orientations on nanoparticles by straightforward use of the equations derived in Stöhr and Outka's paper [11]. In order to realize the capability of NEXAFS to probe molecular orientations on nanoparticles, an understanding of how adsorbates on different parts of a nanoparticle contribute is required.

Here we have simulated NEXAFS measurements on a hypothetical sample that consists of a single CO-covered nanoparticle. For argument's sake, we specify a $\mathrm{Pd}$ nanoparticle supported on $\mathrm{TiO}_{2}(110)$ simply so that we can set the general particle shape as a truncated cuboctahedron, in line with experimental observations [21]. Using these particles, we examine the influence of the particle size and shape, as well as the $\mathrm{CO}$ orientation on the CO $\pi^{*}$ NEXAFS polarization dependence. Two different experimental geometries were used. In the first geometry, the incident photon beam was set to be $p$ polarized, i.e., with its electric-field vector $\mathbf{E}$ lying in the plane of incidence (POI), the $\pi^{*}$ resonance signal from the (CO-covered) nanoparticle was then calculated as a function of the incidence angle. This geometry is the standard geometry as used in connection with a conventional synchrotron radiation source. In the second geometry, which represents a typical XPEEM experiment with double undulator to rotate the electric field vector of the X-rays, the angle of incidence was set at a grazing angle of $16^{\circ}$. The $\pi^{*}$ resonance signal was calculated as a function of the polarization angle, $\beta$, which governs the direction of $\mathbf{E}$ relative to POI. In doing so, a systematic study of the effects of the aforementioned factors upon the angular dependence of the $\pi^{*}$ resonance intensity from the nanoparticle can be achieved. There are experimental data available for the second geometry. These are compared with the simulations.

\section{Methodology}

\subsection{Defining the Shape and Size of a Nanoparticle}

Here we simulate the angular dependence of the CO $\pi^{*}$ NEXAFS from $\mathrm{CO}$ on a hypothetical well-defined $\mathrm{Pd}$ nanoparticle. The nanoparticle comprises a (111) top facet with $\{111\}$ and $\{100\}$ side facets. The exact geometry of the nanoparticle is defined by a set of parameters $(M, m$, $s)$ where $M$ is the number of atoms along the edge between the (111) top facet and one of the $\{111\}$ side facets, $m$ is the number of atoms along the edge between the (111) top facet and $\{100\}$ one of the side facets, and $s$ is the number of (111) layers in the particle. Equation (1) can be used to calculate the number of atoms, $n_{j}$, on different parts of the nanoparticle (where $j=0$ corresponds to the (111) top facet, $j=1-3$ corresponds to each of the $\{111\}$ side facets, $j=4-6$ each of the $\{100\}$ side facets, $j=7-9$ each of the edges between the (111) top facet and one of the $\{100\}$ side facets, $j=10-12$ each of the edges between the (111) top facet and one of the $\{100\}$ side facets, $j=13-18$ each of the edges between a $\{111\}$ and $\{100\}$ side facet, and $j=19-24$ each of the corner sites). In the case of the facets, the associated edge and corner atoms are not included so as not to double count.

$$
n_{j}=\left\{\begin{array}{cc}
\left(M^{2}+m^{2}+4 M m-9 M-9 m+14\right) / 2, & j=0 \\
\left(s^{2}+2 M s-2 M-5 s+4\right) / 2, & 1 \leq j \leq 3 \\
m s-m-2 s+2 & 4 \leq j \leq 6 \\
M-2 & 7 \leq j \leq 9 \\
m-2 & 10 \leq j \leq 12 \\
s-1 & 13 \leq j \leq 18 \\
1 & 19 \leq j \leq 24
\end{array}\right.
$$

An example of such a nanoparticle is shown in Fig. 1a. The particle has $(M, m, s)=(4,3,3)$. Hence, it has 12 atoms in the (111) top facet, 21 atoms in the $\{111\}$ side facets, 6 atoms in the $\{100\}$ side facets, and a total of 27 atoms on the edge (orange) and corner sites (red). To simulate the NEXAFS angular dependence, we have assumed that all parts of the nanoparticle are covered with 0.5 monolayer (ML) of CO [22, 23]. With this assumption, the number of $\mathrm{CO}$ molecules on each part of the particle depends only on the particle geometry, i.e. the number of atoms in each facet, edge or corner.

\subsection{Normal Vectors of Different Facets, Edges and Corners}

To understand how $\mathrm{CO}$ molecules on different parts of the nanoparticle contribute to the X-ray absorption signal, we first need to know how the $\mathrm{CO}$ molecules are oriented 


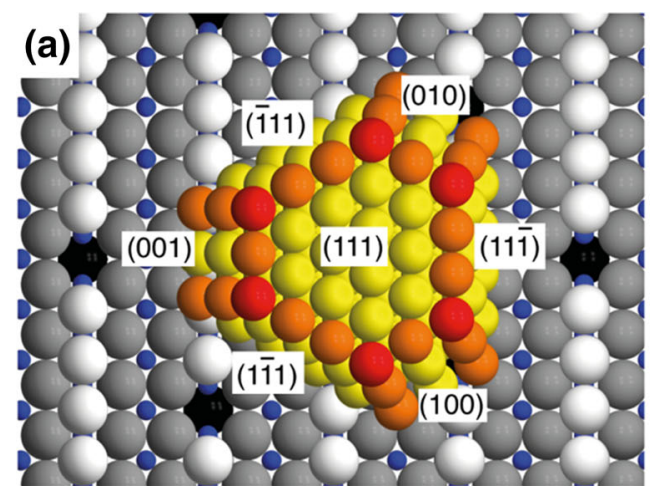

Fig. 1 a Ball model of a hypothetical Pd nanoparticle supported on $\mathrm{TiO}_{2}(110)$. The particle is defined as a (111) top facet with $\{111\}$ and $\{100\}$ side facets. The particle was constructed using parameters $(M$, $m, s)=(4,3,3)$, where $M$ and $m$ are the number of atoms along the edge between the (111) top facet and one of the $\{111\}$ side facets, and one of the $\{100\}$ side facets, respectively, and $s$ is the number of (111) layers in the particle. b The coordinate system employed in the

(relative to the incident photon beam). Even in the simplest case where $\mathrm{CO}$ is assumed to bond with its molecular axis parallel to the normal of the facet, edge or corner, there are many normal vectors.

The normal vectors of facets are straightforward to find: for cubic crystals, facets defined as $(h k l)$ have their normal vectors pointing in the $[\mathrm{hkl}]$ direction [24] so that the (111) top facet will have a normal vector pointing [111] in the direction, while that of the (111) side facet will point in the [111] direction. Once the magnitude of the vectors are made equal, the normal vector for edges and corner sites can be determined by vector addition of the normal vectors from the two facets that make the edge, and the three facets that make the corner, respectively. Thus, the normal vectors for edges between the (111) top facet and the $\{111\}$ side facets are in the $\langle 110\rangle$ directions, those for edges between the (111) top facet and the $\{100\}$ side facets are in the $\left\langle\begin{array}{lll}1+\sqrt{3} & 1 & 1\rangle \text { directions, those for edges }\end{array}\right.$ between the $\{111\}$ side facets and $\{100\}$ side facets are in the $\left\langle\begin{array}{lll}1+\sqrt{3} & \overline{1} & 1\rangle \text { directions, and those for corner sites }\end{array}\right.$ are in the $\langle 2+\sqrt{3} \quad 0 \quad 2\rangle$ directions.

In this way, the normal vectors of all facets, edges and corner sites of the particle $\left(\mathbf{N}_{\mathbf{j}}\right.$, for all $\left.j\right)$ can be determined. In cases where $\mathrm{CO}$ is modeled with a bond orientation away from the facet (edge or corner) normal, the $\mathrm{CO}$ bond vector, $\mathbf{B}_{\mathbf{C O}, \mathbf{j}}$, is referenced to the normal vector of the facet (edge or corner) that it is adsorbed on.

\subsection{Orientation of the Nanoparticle and the Incident Photon Beam}

Figure $1 \mathrm{~b}$ displays the coordinate system employed in the simulation. The vector $\mathbf{N}_{\text {particle }}=\left(1, \theta_{\text {particle }}, \phi_{\text {particle }}\right)$ (in

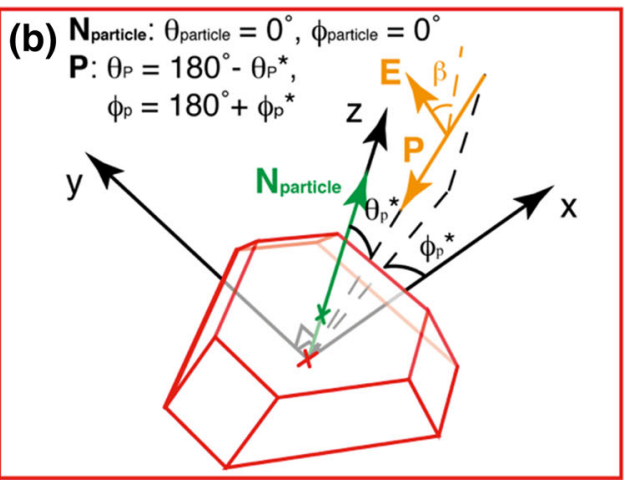

simulation. The vectors $\mathbf{N}_{\text {particle }}=\left(1, \theta_{\text {particle }}, \phi_{\text {particle }}\right)$ and $\mathbf{P}=(1$, $\left.\theta_{P}, \phi_{P}\right)$ denote the orientation of the nanoparticle and the direction of the incident photon beam, respectively. The polarization angle, $\beta$, governs the orientation of the electric field vector $\mathbf{E}$ relative to the plane of incidence. When $\beta=90^{\circ}\left(0^{\circ}\right)$, the incidence photon beam is $s^{-}(p-)$ polarized

spherical form) denotes the orientation of the nanoparticle. Note that $\mathbf{N}_{\text {particle }}$ points in the same direction as the normal vector of the (111) top facet so that when $\mathbf{N}_{\text {particle }}$ $=\left(1,0^{\circ}, 0^{\circ}\right)$, the particle is oriented with the normal vector of its (111) top facet pointing in the $z$-direction and with the normal vector of its (11) side facet (Fig. 1a) lying in the $x z$-plane. If the particle is rotated (counterclockwise) by an angle $\phi$ about the $z$-direction, then $\mathbf{N}_{\text {particle }}=\left(1,0^{\circ}, \phi\right)$ and all the facet (edge and corner) normal vectors, $\mathbf{N}_{\mathbf{j}}$ (for all $j$ ) and hence all the corresponding $\mathrm{CO}$ bond-vectors, $\mathbf{B}_{\mathbf{C O}, \mathbf{j}}$, rotate correspondingly.

$\mathbf{P}=\left(1, \theta_{P}, \phi_{P}\right)$ defines the direction of the incident photon beam. When the nanoparticle is oriented with its top facet facing towards the positive $z$-direction, $\mathbf{P}=\left(1,180^{\circ}\right.$, $180^{\circ}$ ) represents normal incidence while $\mathbf{P}=\left(1,90^{\circ}, 180^{\circ}\right)$ represents in-plane incidence so that $\mathbf{P}=\left(1,106^{\circ}, 180^{\circ}\right)$ corresponds to grazing incidence $16^{\circ}$ away from the surface. Here we assume that the incident photon beam is linearly polarized and an angle, $\beta$ (known as the polarization angle), measured from POI defines the orientation of the electric field vector $\mathbf{E}$ of the incident photon beam relative to POI. Thus, when $\beta=90^{\circ}\left(0^{\circ}\right)$, the light is $s$ - $(p-)$ polarized.

\subsection{Photon Dose and CO $\pi^{*}$ Transition Yield}

The photon dose on part $j$ of the nanoparticle, $D_{j}$, is equal to the absolute value of the scalar product of the corresponding normal vector, $\mathbf{N}_{\mathbf{j}}$, and the incident photon beam, P:

$D_{j}=\left|\mathbf{N}_{j} \cdot \mathbf{P}\right|$

We take the absolute value $D_{j}$ here because at photon energies between 280 and $320 \mathrm{eV}$, the incident photon beam can penetrate through nanoparticles with average diameters 
less than $20 \mathrm{~nm}$ and photo-excite adsorbates (in this case, $\mathrm{CO}$ ) that are not in line-of-sight. Using the tabulated results in Henke et al. [25], the X-ray attenuation length in $\mathrm{Pd}$ at $h v=300 \mathrm{eV}$ is calculated to be $151.4 \mathrm{~nm}$. Hence, the percentage of $\mathrm{X}$-rays that can transmit through a $\mathrm{Pd}$ nanoparticle with an average diameter of $20 \mathrm{~nm}$ is equal to $e^{-\frac{x}{L}}=e^{-\frac{20}{151.4}}=87.6 \%$. Given that Eq. (2) does not include this X-ray attenuation, the signal contribution of $\mathrm{CO}$ not in line-of-sight to the photon beam must be over-estimated. However, as long as the nanoparticle does not exceed $20 \mathrm{~nm}$ in diameter, our method should nevertheless provide a reasonable, qualitative prediction of the experimental results.

We also need to understand how a single $\mathrm{CO}$ molecule interacts with the incoming photon beam. Since $\mathrm{CO}$ is a multiply-bonded diatomic molecule, its $\pi^{*}$ orbital is a plane-orbital whose normal vector $\left(\mathbf{O}_{\mathbf{C O}}\right)$ is parallel to the intra-molecular bond vector of $\mathrm{CO}\left(\mathbf{B}_{\mathbf{C O}}\right)$. According to the dipole approximation [11], the experimental cross section of the $1 \mathrm{~s} \rightarrow \pi^{*}$ transition of a $\mathrm{CO}$ molecule at a facet (edge or corner) $j, I_{C O}, j$, is proportional to one minus the square of the dot product between the electric field vector $\mathbf{E}$ and the intra-molecular bond vector of the $\mathrm{CO}$ molecule, and has the following form:

$I_{C O, j}=A\left(1-\left|\mathbf{E} \cdot \mathbf{B}_{\mathbf{C O}, \mathbf{j}}\right|^{2}\right)$

where $A$ is a constant, $\mathbf{B}_{\mathbf{C O}}, \mathbf{j}$ the intra-molecular bond vector of the $\mathrm{CO}$ at facet (edge or corner) $j$.

\subsection{Effect of the Rotational Symmetry of the Facet}

In the case where a $\mathrm{CO}$ molecule is considered to bond with an angle away from the normal vector of facet $j$, the angular dependence of the $\pi^{*}$ transition yield as depicted in Eq. (3) depends on the azimuthal orientation of the molecule relative to the facet. In many systems of adsorbates on semiinfinite surfaces, this azimuthal dependence is eliminated by the substrate symmetry [11]. Here, we take the effect of facet-symmetry into account by considering the equivalent bonding orientations of $\mathrm{CO}$ on different facets, arising from facet-symmetry. For instance, on each of the $\{111\}$ facets, each $\mathrm{CO}$ can bond in three equivalent directions, separated by $120^{\circ}$ from each other about the azimuthal axis, due to the threefold rotation symmetry of the $\{111\}$ facet. Similarly, on each of the $\{100\}$ side facets each $\mathrm{CO}$ can bond in four equivalent directions, separated by $90^{\circ}$ from each other about the azimuthal axis, arising from the fourfold rotation symmetry of the $\{100\}$ side facet. Equation (3) can therefore be modified as follows:

$I_{C O, j}=\frac{A}{R_{j}} \sum_{1 \leq k \leq R_{j}}\left(1-\left|\mathbf{E} \cdot \mathbf{B}_{\mathbf{C O}, \mathbf{j}, \mathbf{k}}\right|^{2}\right)$ where $R_{j}$ denotes the number of equivalent bond orientations of CO (and hence the $R$-fold rotation symmetry) on facet (edge or corner) $j$. Then, the $\pi^{*}$ resonance intensity arising from all $\mathrm{CO}$ on facet (edge or corner) $j, I_{j}$, is equal to the product of the number of $\mathrm{CO}$ molecules $\left(n_{j} \times \theta_{C O}\right)$, the photon dose, $D_{j}$, and the (effective) $\pi^{*}$ resonance intensity per $\mathrm{CO}, I_{C O, j}$, on facet $j$, and has the following form:

$I_{j}=n_{j} \times \theta_{C O} \times D_{j} \times I_{C O, j}$

Then, by summing the $\pi^{*}$ resonance intensities from all parts of the nanoparticle, we have the total $\pi^{*}$ resonance intensity as follows:

$I_{\text {total }}=\sum_{j} I_{j}$

As the total $\mathrm{CO} \pi^{*}$ resonance intensity from a particle depends greatly on the particle dimension, to facilitate a qualitative comparison of the angular dependence between the $\pi^{*}$ resonance curves of different particles, we normalize the total $\pi^{*}$ resonance intensity to the total number of surface atoms of a particle as follow

$I_{\text {normalized }}=\frac{I_{\text {total }}}{n_{\text {total }}}$ or $\frac{\sum_{j} I_{j}}{\sum_{j} n_{j}}$

\section{Results and Discussions}

In carbon K-edge NEXAFS measurements, the bonding orientation of $\mathrm{CO}$ on a surface can be probed by measuring the integrated intensity of the $\mathrm{CO} \pi^{*}$ resonance peak (located at $h v=287 \mathrm{eV}$ ) as a function of either the angle of incidence, $\theta$ (with the incident light oriented to be $p$-polarised) [11], or as a function of the direction of the electric field vector $(\mathbf{E})$ of the incident photon beam, hence the polarization angle, $\beta$ [26]. The former method gives $I$ versus $\theta$ curves while the latter method leads to $I$ versus $\beta$ curves. We will treat these separately. In part one, we will present simulated $I$ versus $\theta$ curves of nanoparticles and factors that can influence its angular dependence. In part two, we will discuss simulated $I$ versus $\beta$ curves.

As mentioned previously, all the hypothetical particle consists of a (111) top facet as well as $\{111\}$ and $\{100\}$ side facets, edges and corner sites. In order to study the influence of the particle geometry on the overall $I$ versus $\theta$ curve, we first define three terms: $R_{T S}, R_{S S}$, and $R_{d}$, where $R_{T S}$ corresponds to the ratio between the number of surface atoms in the (111) top facet and those on the side facets, $R_{S S}$ is the ratio between the number of atoms in the $\{111\}$ 
side facets and those on the $\{100\}$ side facets, and $R_{d}$ is the number percentage of surface atoms on the particle that are at the edge and corner sites. By varying one term at a time, the effect of particle geometry can be investigated systematically.

\subsection{Versus $\theta$ Curve}

\subsubsection{Individual I Versus $\theta$ Curves from Different Parts of the Hypothetical Nanoparticle}

Figure 2 shows the simulated $\mathrm{CO} \pi^{*}$ resonance peak intensity versus angle of incidence ( $I$ vs $\theta$ ) curves obtained from different parts of a CO-covered nanoparticle. The particle was constructed using parameters $(M, m, s)=(12$, $8,4)$ and has an average diameter of $5.1 \mathrm{~nm}$ and a height of $0.81 \mathrm{~nm}$. In the simulation, the incident photon beam $(\mathbf{P})$ is linearly, $p$-polarized (hence $\beta=0^{\circ}$ ) with its direction always fixed at $\mathbf{P}=\left(1, \theta_{P}, \phi_{P}\right)=\left(1,180^{\circ}, 180^{\circ}\right)$. To simplify the picture, we assume that $\mathrm{CO}$ bonds with its molecular axis parallel to the corresponding facet (edge or corner) normal, hence $\mathbf{B}_{\mathbf{j}}=\mathbf{N}_{\mathbf{j}}$ for all $j$. Also, in order to better illustrate the contributions from different parts of the particle, we rotate the azimuth of the particle by $5^{\circ}$, i.e. $\mathrm{N}_{\text {particle }}=\left(1,0,5^{\circ}\right)$. This removes the equivalence of the side facets, edges and corners relative to the incident light that arises from the mirror symmetry of the system, as shown in Fig. 1b. All the facet (edge and corner) normal vectors as well as the corresponding $\mathrm{CO}$ bond vectors will rotate correspondingly. As shown in Fig. 2, due to the different orientations of $\mathrm{CO}$ relative to the incident photon beam at different parts of the particle, the $I$ versus $\theta$ curves are clearly different from each other. The individual $I$ versus $\theta$ curves can vary depending upon the bond orientation of $\mathrm{CO}\left(\mathbf{B}_{\mathbf{j}}\right)$ on the corresponding facet, edge or corner, the orientation of the particle relative to the incident photon beam $(\mathbf{P})$, and the direction of the electric field vector $\mathbf{E}$ of the incident photon beam (hence the polarization angle $\beta$ ).

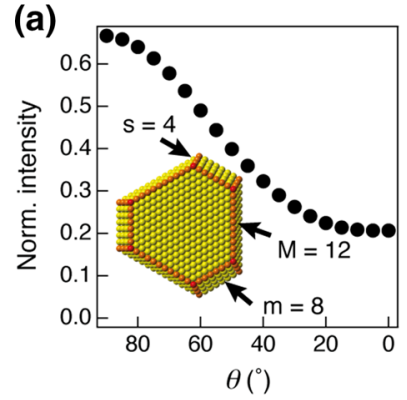

(e)

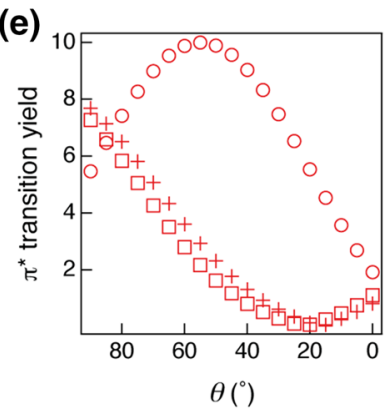

Edges between (111) top facet \& $\{111\}$ side facets

$\circ(1 \overline{1} 1) \square(\overline{1} 11)+(11 \overline{1})$ (b)

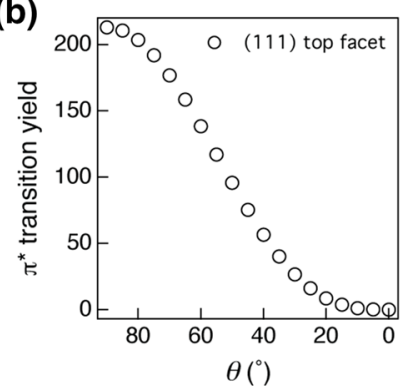

(f)

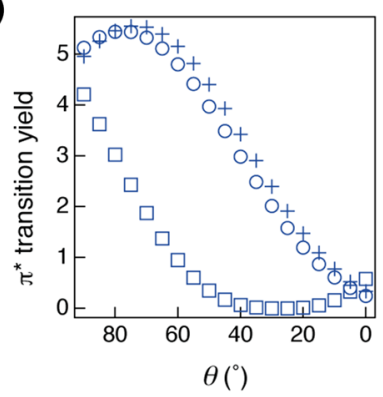

Edges between (111) top facet \& $\{100\}$ side facets

$\circ(010) \square(001)+(100)$ (c)

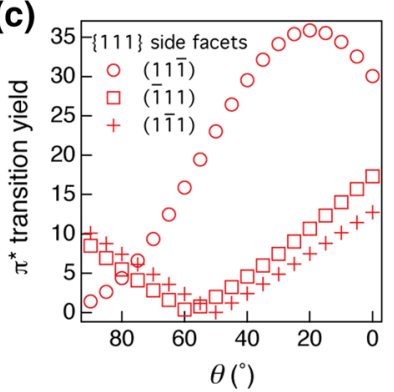

(g)

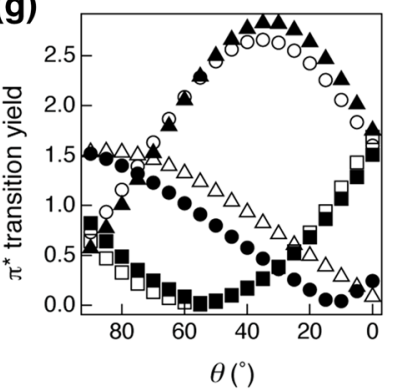

Edges between $\{111\} \&\{100\}$ side facets O $(11 \overline{1}) \&(010) \quad(\overline{1} 11) \&(010)$ $\square(\overline{1} 11) \&(001) \quad(1 \overline{1} 1) \&(001)$ $\triangle(1 \overline{1} 1) \&(100) \quad \Delta(11 \overline{1}) \&(100)$
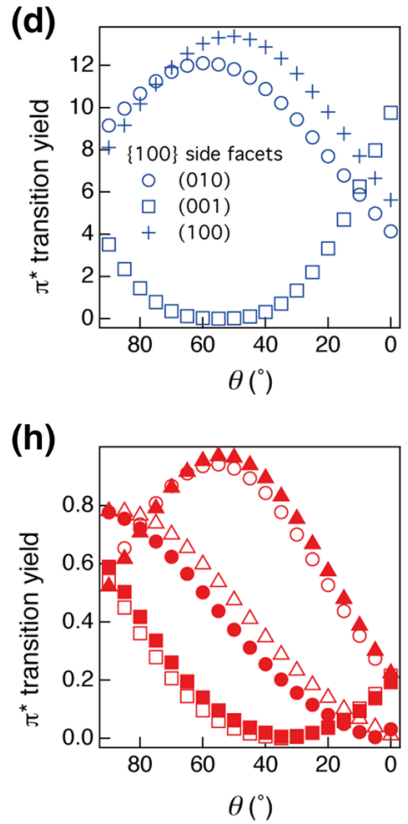

Corners between (111) top facet, $\{111\}$ $\&\{100\}$ side facets

○ $(11 \overline{1}) \&(010) \quad(\overline{1} 11) \&(010)$

$\square(\overline{1} 11) \&(001) \quad \square(1 \overline{1} 1) \&(001)$

$\triangle(1 \overline{1} 1) \&(100) \quad \Delta(11 \overline{1}) \&(100)$

Fig. 2 Simulated $\pi^{*}$ resonance intensity versus angle of incidence $(I$ versus $\theta$ ) curves obtained from different parts of a CO-covered nanoparticle constructed using parameters $(M, m, s)=(12,8,4)$. a The total curve that is a summation over curves from different parts of the particle (illustrated in the inset) including: b (111) top facet, c $\{111\}$ side facets, d $\{100\}$ side facets, e edges between the (111) top and $\{111\}$ side facets, $\mathbf{f}$ edges between the (111) top and $\{100\}$ side facets, $\mathbf{g}$ edges between $\{111\}$ and $\{100\}$ side facets, and h corners sites. After summation, the total curve in (a) is then normalized to the number of surface atoms of the particle. The

direction of the incident photon beam is fixed at $\mathbf{P}=\left(1,180^{\circ}, 180^{\circ}\right)$. The incident light is set to be completely $p$-polarized, hence $\beta=0^{\circ}$. $\mathrm{CO}$ on different parts of the particle is set to bond with their molecular axes parallel to the corresponding facet (edge or corner) normal, hence $\mathbf{B}_{\mathbf{j}}=\mathbf{N}_{\mathbf{j}}$ for all $j$. In order to show the contributions from all facets, edges and corner sites more effectively, the particle is deliberately rotated about its azimuth by $5^{\circ}$, hence $\mathbf{N}_{\text {particle }}\left(1,0^{\circ}, 5^{\circ}\right)$. This breaks the mirror symmetry of the nanoparticle about the plane of incidence, removing the equivalence of different pairs of facets, edges and corners relative to the incident light 
The overall $I$ versus $\theta$ curve displayed in Fig. $2 \mathrm{a}$ is a sum over all $I$ versus $\theta$ curves from different parts of the particle, which is then divided by the total number of surface atoms on the particle. As we assume that the $\mathrm{CO}$ coverage is uniform across the particle, the angular dependence of the overall $I$ versus $\theta$ curve depends directly on the particle geometry.

\subsubsection{Particle Size Effect}

To investigate the effect of the particle size, we fixed $R_{T S}$ at 0.8 and $R_{S S}$ at 0.2 . With these values fixed, the only effect of reducing the particle size is an increase in the proportion of edge and corner sites relative to the top and side facets of the particle, i.e. only $R_{d}$ varies. Figure 3 displays the simulated $I$ versus $\theta$ curves for a series of such particles. For a particle (particle A, average diameter $(d)=16.7 \mathrm{~nm}$, height $(h)=3.7 \mathrm{~nm}$ ) with an $R_{d}$ value of $4.6 \%$, the corresponding $I$ versus $\theta$ curve (open circles) starts with an intensity of 0.58 at $\theta=90^{\circ}$ (normal incidence), then decreases monotonically (with decreasing $\theta$ ) and reaches an intensity of 0.27 at $\theta=0^{\circ}$. Reducing the particle size only leads to a small change to the overall $I$ versus $\theta$ curve. For a particle (particle D, $d=1.5 \mathrm{~nm}, h=0.36 \mathrm{~nm}$ ) with an $R_{d}$ value of $53 \%$, the corresponding $I$ versus $\theta$ curve (crosses) starts with an intensity of 0.57 at normal incidence, then drops at a slower rate at $\theta>60^{\circ}$, below which it decreases faster and reaches a lower intensity of 0.24 at $\theta=0^{\circ}$. On this basis, we summarize that if $R_{d}<20 \%$, $\mathrm{CO}$ molecules on edge and corner sites of the particle have only a small effect on the overall $I$ versus $\theta$ curve.

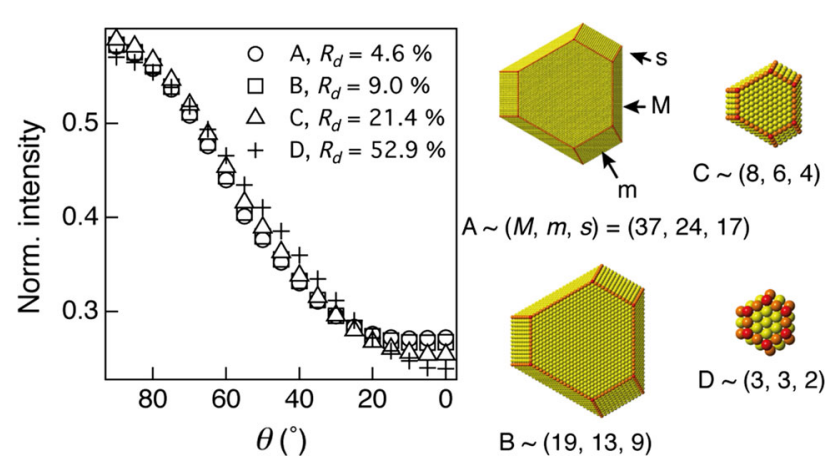

Fig. 3 Simulated $I$ versus $\theta$ curves obtained from CO-covered Pd nanoparticles that are constructed using different sets of parameters. $(M, m, s)=(37,24,17)$ for particle A (circles),$(19,13,9)$ for particle B (squares), $(8,6,4)$ for particle C (triangles), and $(3,3,2)$ for particle $\mathrm{D}$ (pluses). All particles have the same $R_{T S}=0.8$ and $R_{S S}=2.0$ but varying $R_{d}$. On this basis, any difference between the simulated $I$ versus $\theta$ curves can be attributed to the particle size effect. The particle is oriented as in Fig. 1b. CO is set to bond with its molecular axis parallel to the facet normal, hence $\mathbf{B}_{\mathbf{j}}=\mathbf{N}_{\mathbf{j}}$ for all $j$. The direction of the incident photon beam is fixed at $\mathbf{P}=\left(1,180^{\circ}\right.$, $\left.180^{\circ}\right)$. The incident light is set to be $p$-polarized, hence $\beta=0^{\circ}$

\subsubsection{Relative Size of Side Facets}

We can investigate the effect of different proportions of side facets by varying $R_{S S}$ with $R_{T S}$ fixed. Due to the differing orientation of $\mathrm{CO}$ on different facets, $\mathrm{CO}$ on each facet interacts differently with the incident light. In all the particles simulated in this section, $R_{d}<15 \%$ so that the signal contribution from $\mathrm{CO}$ on the edge and corner sites is negligible (Fig. 3). In addition, the particles have average diameters less than $20 \mathrm{~nm}$ so we assume that there is total $\mathrm{X}$-ray transmission through the nanoparticles. In this way, any differences in the simulated $I$ versus $\theta$ curves can be attributed to a change in the ratio of the side facets of the nanoparticle. The simulation results, together with the shapes of the simulated particles are displayed in Fig. 4. We start with $I$ versus $\theta$ curves obtained from particles with the same $R_{T S}$ value of 3.2 (Fig. 4a). At this high $R_{T S}$ value, $\mathrm{CO}$ on the (111) top facet always contributes most to the total X-ray absorption signal and hence changing the relative portions of the $\{111\}$ and $\{100\}$ side facets from one extreme $\left(R_{S S}=35.5\right)$ to another $\left(R_{S S}=0.07\right)$ only leads to a minute change in the $I$ versus $\theta$ curve.

With particles with an equal proportion of atoms in the top and side facets, changing the ratio of the side facets should have a much more noticeable effect on the $I$ versus $\theta$ curves. As shown in Fig. $4 \mathrm{~b}$, with $R_{T S}=1$, varying $R_{S S}$ from one extreme (37.5) to another (0.13) causes the $I$ versus $\theta$ curve to drop at an increasing rate with decreasing $\theta$ and reach a lower intensity value at $\theta=0^{\circ}$. The change in the $I$ versus $\theta$ curve with $R_{S S}$ becomes even more prominent at a low value of $R_{T S}=0.22$. As shown in Fig. 4c, while the $I$ versus $\theta$ curve obtained from a crystal having $R_{S S}=0.38$ still exhibits a monotonic decrease with decreasing $\theta$, those from the particles having higher $R_{S S}$ values are markedly different in shape: with $R_{S S}=1$, the $\pi^{*}$ resonance intensity does not vary with $\theta$ at $\theta<55^{\circ}$, and at $R_{S S}=46$, the $I$ versus $\theta$ value first drops in intensity with decreasing $\theta$ at $\theta>55^{\circ}$, below which it increases to reach an intensity value at $\theta=0^{\circ}$ that is higher than the intensity at normal incidence.

\subsubsection{Relative Size of the Top Facet}

We have run simulations for particles that have a fixed $R_{S S}$ value but different $R_{T S}$ values. The simulation results along with the shapes of some of the simulated particles are illustrated in Fig. 5. We first discuss the $I$ versus $\theta$ curves obtained from particles that have a large $R_{S S}$ value of 35 and almost all (97\%) their side facet atoms in the $\{111\}$ orientation. As a result, reducing the $R_{T S}$ value will only increase the signal contribution from the $\{111\}$ side facets relative to that from the (111) top facet. As shown in Fig. 5a, for a particle having a $R_{T S}$ value of $6.48, \mathrm{CO}$ on the 
(a)
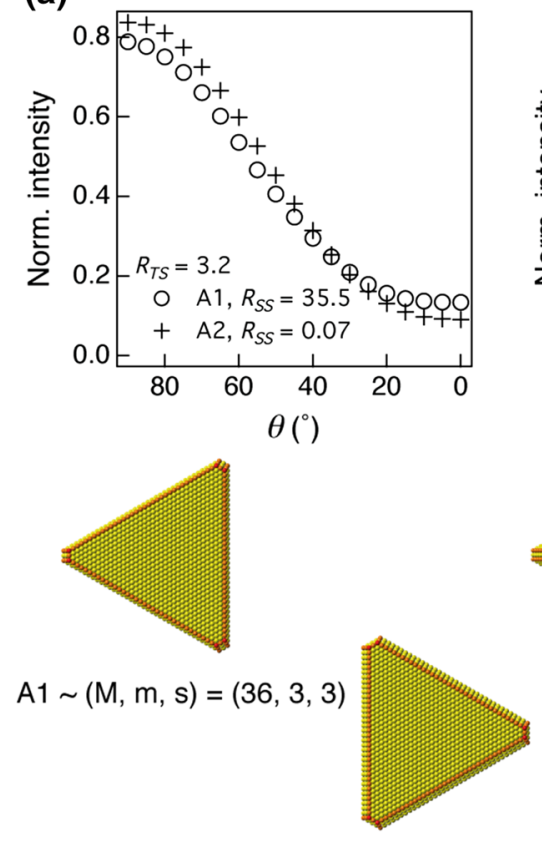

$\mathrm{A} 2 \sim(3,36,3)$ (b)
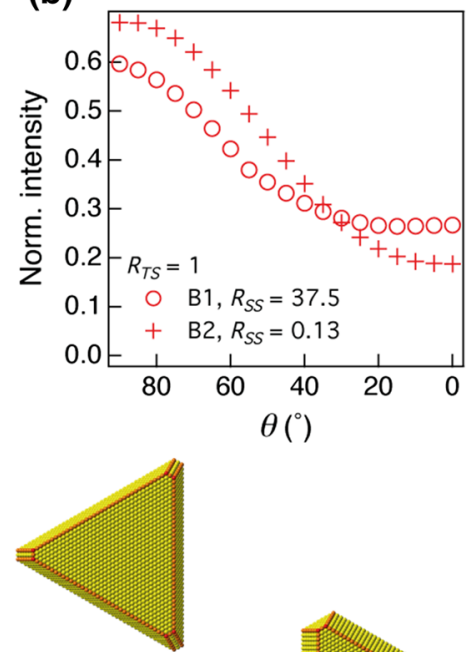

$\mathrm{B} 1 \sim(36,3,7)$

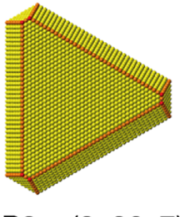

$\mathrm{B} 2 \sim(3,36,7)$ (c)
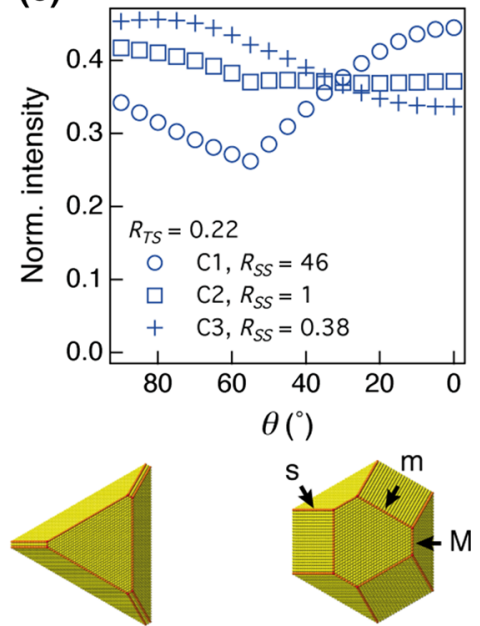

$\mathrm{C} 1 \sim(36,3,24)$

$\mathrm{C} 2 \sim(10,22,24)$

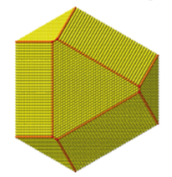

C3 $(3,36,24)$

Fig. 4 Simulated $I$ versus $\theta$ curves obtained from $\mathrm{CO}$ covered nanoparticles having the same $R_{T S}$ value but different $R_{S S}$ values: a $R_{T S}=3.2$ with $R_{S S}$ varying between 35.5 and 0.07 ; $\mathbf{b} R_{T S}=1$ with $R_{S S}$ varying between 37.5 and 0.13 ; $\mathbf{c} R_{T S}=0.22$ with $R_{S S}$ varying between 46 and 0.38 . All the simulated particles have average diameters of $<18 \mathrm{~nm}$ and height of $<8 \mathrm{~nm}$, and have $<15 \%$ of their surface atoms located at the edge and corner sites. As a result, the

(111) top facet contributes most to the total X-ray absorption signal and hence the corresponding $I$ versus $\theta$ curve (open circles) exhibits a strong dependence on $\theta$ and reaches an intensity offset of 0.082 at $\theta=0^{\circ}$. Apart from the non-zero intensity offset at $\theta=0^{\circ}$, this curve resembles that from $\mathrm{CO}$ on a $\mathrm{Pd}(111)$ single crystal where $\mathrm{CO}$ bonds upright on the surface [27]. As $R_{T S}$ decreases, the $I$ versus $\theta$ curve has a descending intensity at normal incidence and drops at a slower rate with decreasing $\theta$. However, when $R_{T S}<0.29$, the $I$ versus $\theta$ curve (open rhombus) starts to develop a different character: rather than decreasing monotonically, the curve now has a breakpoint at $\theta=55^{\circ}$, below which it increases to reach a higher intensity value at $\theta=0^{\circ}$. This character becomes more prominent as $R_{T S}$ decreases further. In the extreme case of $R_{T S}=0.001$ (at which the particle has almost all of its surface atoms in the $\{111\}$ side facets), the $I$ versus $\theta$ curve (crosses) has a very low intensity of 0.20 at normal incidence, then decreases slightly at $\theta>75^{\circ}$, after which it increases to reach an intensity of 0.54 at $\theta=0^{\circ}$.

Since it is not possible to construct nanoparticles that consist of only $\{100\}$ side facets, when varying $R_{T S}$, we could only construct particles with fixed $R_{S S}$ down to 0.5 . contributions of $\mathrm{CO}$ on those sites become negligible. The particles are oriented as in Fig. 1b. CO is set to bond with its molecular axis parallel to the corresponding facet (edge or corner) normal vector, hence $\mathbf{B}_{\mathbf{j}}=\mathbf{N}_{\mathbf{j}}$ for all $j$. The direction of the incident photon beam is set at $\mathbf{P}=\left(1,180^{\circ}, 180^{\circ}\right)$. The incident light was set to be $p$ polarized, hence $\beta=0^{\circ}$. Each particle is illustrated under its respective curve

At $R_{S S}=0.5$, two-thirds of the side facets atoms are in $\{100\}$ facets so any observable change in the simulated $I$ versus $\theta$ curve originates mainly from the varying contribution of the $\{100\}$ side facets. The results are shown in Fig. $5 \mathrm{~b}$. For a particle with a large $R_{T S}$ value of $7.9, \mathrm{CO}$ on the (111) top facet contributes most to the total X-ray absorption signal and hence the corresponding $I$ versus $\theta$ curve (open-circles) resembles that from $\mathrm{CO}$ on a $\mathrm{Pd}(111)$ single crystal surface [27]. As $R_{T S}$ decreases, while still retaining the same essential character, the $I$ versus $\theta$ curve exhibits a diminishing dependence on $\theta$ so that for a particle with $R_{T S}=0.14$ (at which the particle has $84 \%$ of its surface atoms in the side facets), the corresponding $I$ versus $\theta$ curve (crosses) is almost invariant with $\theta$.

By comparing the simulation results in Fig. 5a, b, $\mathrm{CO}$ on \{111\} side facets seems to have larger impact on the $I$ versus $\theta$ than those on $\{100\}$ side facets. This can be explained by the larger angular separation between the (111) top facet and each of the $\{111\}$ side facets than that between the (111) top facet and each of the $\{100\}$ side facets. In addition, both Fig. 5a, b reveal that with fixed $R_{S S}$, there exists an incidence angle $(\theta)$ (namely critical $\theta$, or $\theta_{\text {critical }}$ ) at which the signal contributions of $\mathrm{CO}$ on the 


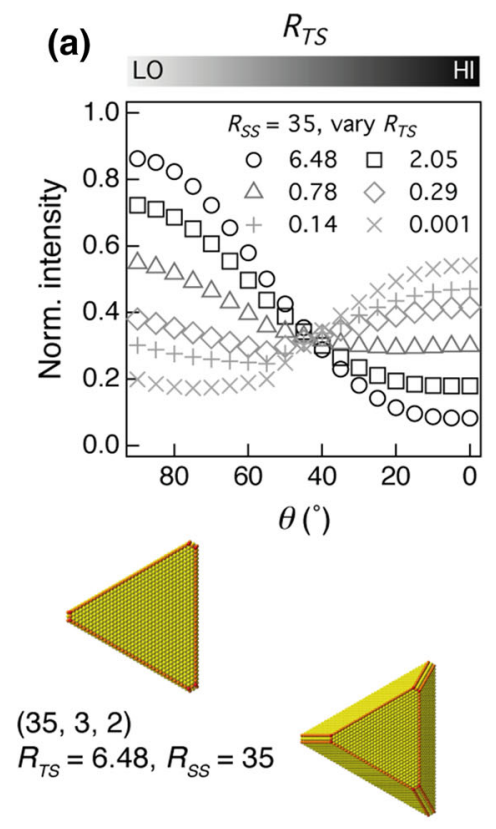

$(29,3,16)$

$R_{T S}=0.29, R_{S S}=35$

Fig. 5 Simulated $I$ versus $\theta$ curves obtained from CO-covered nanoparticles having the same $R_{S S}$ value but different $R_{T S}$ values: a $R_{S S}=35$ with $R_{T S}$ varying between 6.5 and $0.001 ; \mathbf{b} R_{S S}=0.5$ with $R_{T S}$ varying between 7.9 and 0.14 ; c $R_{S S}=1$ with $R_{T S}$ varying between 7.0 and 0.06 . All the simulated particles have average diameter of $<19 \mathrm{~nm}$ and height of $<15 \mathrm{~nm}$, and have $<15 \%$ of their surface atoms located at the edge and corner sites. As a result, the

(111) top facet and the side facets are equal to each other, and therefore the overall $\pi^{*}$ resonance intensity at $\theta_{\text {critical }}$ is invariant with $R_{T S}$.

Figure 5c displays the simulated $I$ versus $\theta$ curves from particles having a unity $R_{S S}$ value but different $R_{T S}$ values. That $R_{S S}=1$ means that the particles have equal numbers of their surface atoms in the $\{111\}$ and $\{100\}$ side facets. As expected, as $R_{T S}$ decreases, the $I$ versus $\theta$ curve varies in shape to an extent that is between those found in Fig. 5a, b.

\subsubsection{Rotating the Particles}

Rotating the particle about the surface normal can influence the angular dependence of the $I$ versus $\theta$ curve because ultimately this changes the orientation of the $\mathrm{CO}$ bond vectors. Here, we maintain the assumption of $\mathrm{CO}$ bonding upright on all parts of the particle, hence $\mathbf{B}_{\mathbf{j}}=\mathbf{N}_{\mathbf{j}}$ for all $j$.

We first examine $I$ versus $\theta$ curves obtained from particles with an $R_{T S}$ value of 3.2. As pointed out previously, at this high $R_{T S}$ value, $\mathrm{CO}$ on the (111) top facet will contribute most to the total X-ray absorption signal. As shown in Fig. 6a, b, rotating such particles about their normal vectors $\left(\mathbf{N}_{\text {particle }}\right.$ ) does not cause any noticeable change to the corresponding $I$ versus $\theta$ curves. This is in
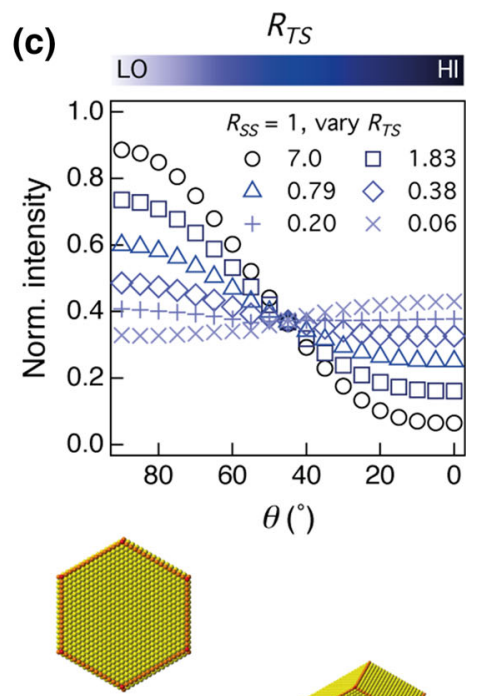

$(15,16,2)$ $R_{T S}=7.0, R_{S S}=1$

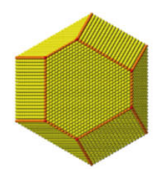

$(12,20,16)$

$(12,30,16)$

$R_{T S}=0.61, R_{S S}=0.5$

$R_{T S}=0.38, R_{S S}=1$

contributions of $\mathrm{CO}$ on those sites become negligible. The particles are oriented in the same manner as shown in Fig. 1b. CO is taken to bond with its molecular axis parallel to the facet normal, hence $\mathbf{B}_{\mathbf{j}}=\mathbf{N}_{\mathbf{j}}$ for all $j$. The direction of the incident photon beam is fixed at $\mathbf{P}=\left(1,180^{\circ}, 180^{\circ}\right)$. The incident light is set to be $p$-polarized, hence $\beta=0^{\circ}$. Some of the particles are illustrated under their respective curves. They can be identified in the graphs by their $R_{T S}$ value

line with expectation because rotating the particle about its normal causes no change to the bond orientation of $\mathrm{CO}$ relative to the incident photon beam, and hence will have no influence upon the X-ray absorption signals.

The next particle we examine has an $R_{T S}$ value of $\sim 0.5$ $(0.51)$ and unity $R_{S S}$. This particle has its (111) top facet as well as $\{111\}$ and $\{100\}$ side facets nearly all of equal size to each other. As displayed in Fig. 6c, due to the increased contributions from the side facets, the $I$ versus $\theta$ curve has a greater dependence on the azimuthal orientation: at $\phi_{\text {particle }}=30^{\circ}$ the $I$ versus $\theta$ curve drops at a lower rate at $\theta>45^{\circ}$ but reaches a smaller intensity at $\theta=0^{\circ}$. At $\phi_{\text {particle }}=60^{\circ}$, although the curve also drops at a lower rate at $\theta>45^{\circ}$, it reaches the same intensity value at $\theta=0^{\circ}$ as the curve obtained at $\phi_{\text {particle }}=0^{\circ}$. At $\phi_{\text {parti- }}$ cle $=90^{\circ}$ the curve is identical to that obtained at $\phi_{\text {parti- }}$ cle $=30^{\circ}$. This is because of the mirror symmetry of the system, with the reflection plane being parallel to the incoming photon beam. It is important to note that this mirror symmetry vanishes when the incident photon beam is not completely $p$ - or $s$ - polarized, i.e. when the electric field vector $\mathbf{E}$ deviates in orientation from the plane of incidence $\left(0^{\circ}<\beta<90^{\circ}\right)$, in which case $\mathrm{CO}$ on different parts of the particle will interact with the incident photon 


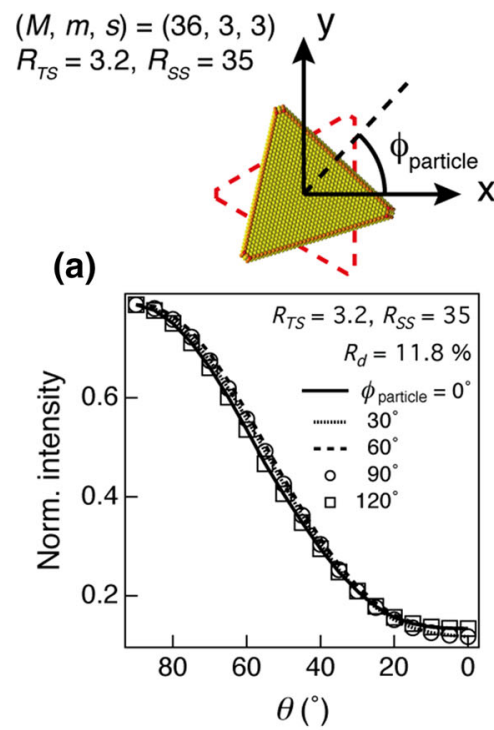

(d)
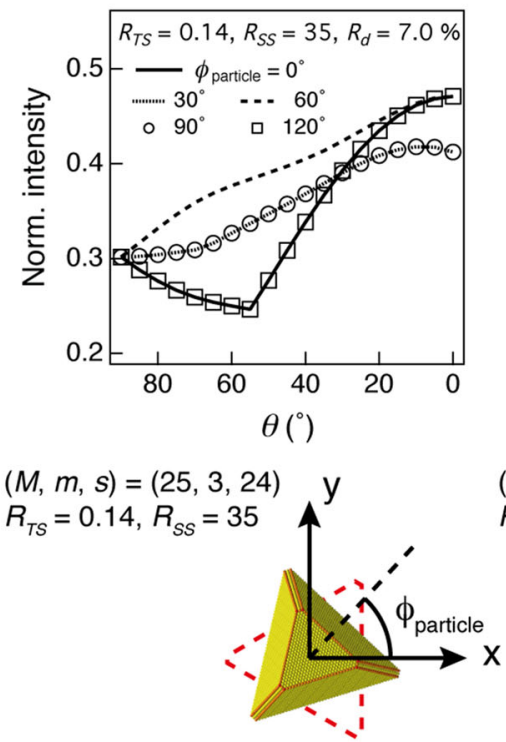

Fig. 6 Simulated $I$ versus $\theta$ curves obtained from CO-covered nanoparticles that are rotated by different angles about their corresponding particle normal vectors. a-b Curves obtained from particles with $R_{T S}=3.2$ and $R_{S S}=35$ (a) and 0.07 (b). c Curves obtained from a particle with $R_{T S}=0.51$ and $R_{S S}=1$. d-f Curves obtained from the particles with $R_{T S}=0.14$ and $R_{S S}$ ranging between 35 and 0.5 . Before azimuthal rotation, the particles are oriented as in

beam differently. At $\phi_{\text {particle }}=120^{\circ}$, the curve is identical to that obtained at $\phi_{\text {particle }}=0^{\circ}$ as a result of the threefold rotation symmetry of the nanoparticle.

We have already shown that rotating the particle about its normal has no effect on the (111) top facet. However, rotating the particle also changes the orientation of all other parts of the particle (and hence the corresponding $\mathrm{CO}$ bond vectors) relative to the incident photon beam. This influences both the photon doses $\left(D_{j}\right)$ as well as the interaction between the electric field vector $\mathbf{E}$ of the incident photon

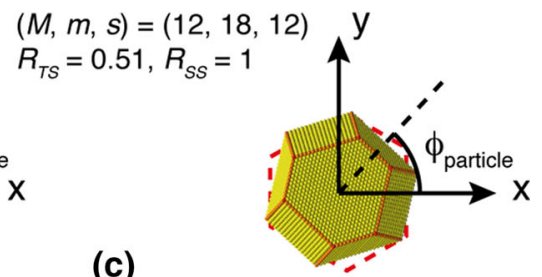

(c)

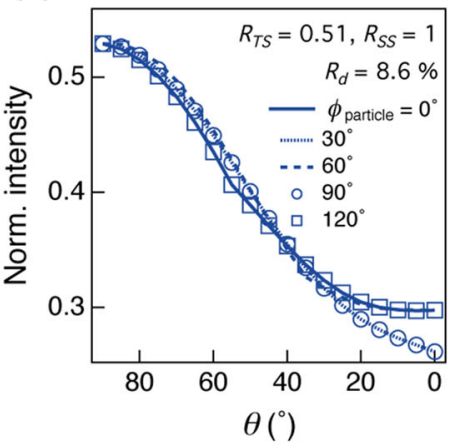

(f)

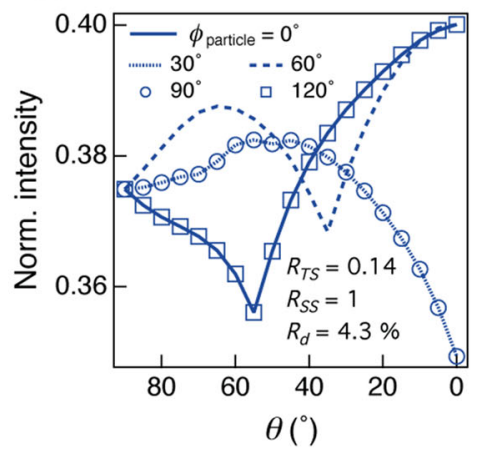

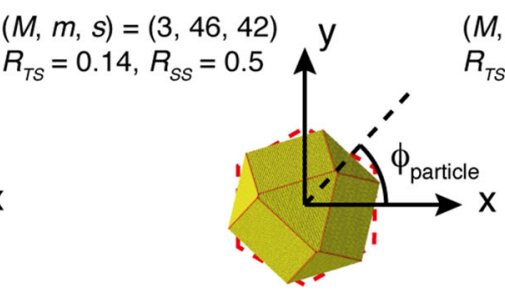

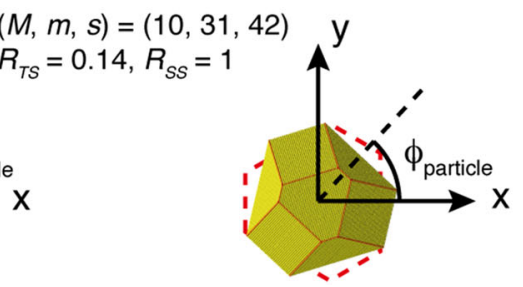

Fig. 1b. CO is assumed to bond with its molecular axis parallel to the corresponding facet (edge or corner) normal vector, hence $\mathbf{B}_{\mathbf{j}}=\mathbf{N}_{\mathbf{j}}$ for all $j$. The direction of the incident photon beam is fixed at $\mathbf{P}=(1$, $180^{\circ}, 180^{\circ}$ ). The incident light is set to be $p$-polarized, hence $\beta=0^{\circ}$. Each particle is illustrated with its respective curves, with its orientation before azimuthal rotation marked with red dashed lines

beam and $\mathrm{CO}$ on different parts (excluding the (111) top facet) of the particle, and as a result, varies the angular dependence of the overall $I$ versus $\theta$ curve.

As the proportion of side facets increases, the $I$ versus $\theta$ curves become more affected by the azimuthal rotation. $I$ versus $\theta$ curves obtained from a particle with $80 \%$ of its surface atoms located in the $\{111\}$ side facets are shown in Fig. 6d. At $\phi_{\text {particle }}=0^{\circ}$, the intensity (solid lines) decreases with $\theta$ to $\theta=55^{\circ}$, below which it increases to reach an intensity of 0.47 at $\theta=0^{\circ}$; at $\phi_{\text {particle }}=30^{\circ}$ 
(dotted lines) the intensity increases steadily with decreasing $\theta$ to $\theta=10^{\circ}$, below which it drops slightly and reaches an intensity of 0.41 at $\theta=0^{\circ}$; at $\phi_{\text {particle }}=60^{\circ}$ (dashed lines) the intensity increases monotonically and reaches an intensity value of 0.47 at $\theta=0^{\circ}$. As before, the identical behavior at $\phi_{\text {particle }}=30^{\circ}$ and $\phi_{\text {particle }}=90^{\circ}$ is due to the system's mirror symmetry, while the identical behavior at $\phi_{\text {particle }}=0^{\circ}$ and $\phi_{\text {particle }}=120^{\circ}$ is due to the threefold rotation symmetry of the particle.

Figure 6e shows $I$ versus $\theta$ curves obtained from a particle having $56 \%$ of its surface atoms located in the $\{100\}$ side facets. This particle has the remaining 28 and $11 \%$ of its surface atoms in the $\{111\}$ side facets and the (111) top facet respectively. Since the $\pi^{*}$ intensity contributions arising from the latter two areas are known, as well by careful inspection it is possible to elucidate how the signal contribution of $\mathrm{CO}$ on the $\{100\}$ side facets is affected by azimuthal rotation.

The variation of the $I$ versus $\theta$ curves with azimuthal rotation is markedly different from that of the particle discussed in Fig. 6d. This is a consequence of the different orientations of the $\{111\}$ and $\{100\}$ side facets; apart from the difference in the angular separation between the (111) top facet and each of the $\{111\}$ side facets $\left(70.5^{\circ}\right)$ and that between the (111) top facet and each of the $\{100\}$ side facets $\left(54.7^{\circ}\right)$, the azimuthal orientation of each of the $\{111\}$ side facets is always separated by $60^{\circ}$ from the adjoining $\{100\}$ side facets.

For a particle having an equal number of its surface atoms in the $\{111\}$ and $\{100\}$ side facets, as the particle rotates, the corresponding $I$ versus $\theta$ curve alters in a way that seems to be a convolution between the changes found in Fig. 6d, e. As before, that the behavior at $\phi_{\text {particle }}=30^{\circ}$ and $90^{\circ}$ and $0^{\circ}$ and $120^{\circ}$ are identical due to the mirror symmetry of the system and the rotational symmetry of the crystal, respectively.

\subsubsection{Changing $\mathrm{C}-\mathrm{O}$ Bond Angle}

We have also calculated how varying the bond orientation of $\mathrm{CO}$ with respect to each of the facet (edge or corner) normal modifies the $I$ versus $\theta$ characteristics. We performed the simulations using two particles with different shapes: the first particle has parameters $(M, m, s)=\left(10^{6}, 3\right.$, 3 ) and has almost all its surface atoms in the (111) top facet, and resembles a $\operatorname{Pd}(111)$ single crystal surface. The second particle has parameters $(M, m, s)=(12,18,12)$ and has almost equal numbers of atoms $(\sim 30 \%)$ located on the (111) top facet, $\{111\}$ side facets, and $\{100\}$ side facets.

Figure 7a illustrates the overall $I$ versus $\theta$ curves obtained from the first particle where $\mathrm{CO}$ is set to bond at different polar angles $\left(\theta_{B}\right)$ away from the corresponding facet (edge or corner) normal (the azimuthal component of the $\mathrm{CO}$ bond vector is fixed at $\phi_{B}=0^{\circ}$ ). As $\theta_{B}$ increases, the $I$ versus $\theta$ curve also deviates in shape: apart from starting with a descending intensity at normal incidence, at $\theta_{B}>30^{\circ}$ the curve also decreases at a rate that increases with decreasing $\theta$. Note that all the curves in Fig. 7a attain a zero intensity at $\theta=0^{\circ}$. This is because at $\theta=0^{\circ}$, the incident photon beam is parallel to the surface, resulting in a negligible photon dose.

With the polar component $\left(\theta_{B}\right)$ of the $\mathrm{CO}$ bond vector fixed at $30^{\circ}$, we tested whether the $I$ versus $\theta$ curve of the first particle is modified by varying the azimuthal component $\left(\phi_{B}\right)$ of the $\mathrm{CO}$ bond vector. As shown in Fig. 7b, the $I$ versus $\theta$ curve is barely affected while varying $\phi_{B}$. This is in good agreement with the case of $\mathrm{CO}$ on the (111) surfaces of fcc crystals [11]: on these systems, any dependence of the $\mathrm{CO} \pi^{*}$ resonance peak intensity on the azimuthal direction of the $\mathrm{CO}$ is eliminated by the threefold rotation symmetry of the substrate.

Varying the bond orientation of $\mathrm{CO}$ modifies the $I$ versus $\theta$ curve of the second particle in a different way. First, as shown in Fig. 7c, not only does increasing the polar component $\left(\theta_{B}\right)$ of the $\mathrm{CO}$ bond vector (with respect to the corresponding facet (edge or corner) normal) lower the $\pi^{*}$ resonance intensity at normal incidence, it also alters the shape of the $I$ versus $\theta$ curve: at $\theta_{B} \geq 30^{\circ}$, the curve develops a double-hump shape that becomes more prominent with increasing $\theta_{B}$. Unlike those in Fig. 7a, the $I$ versus $\theta$ curves here do not reduce to zero intensity at $\theta=0^{\circ}$. This is because at $\theta=0^{\circ}, \mathrm{CO}$ on the side facets on this particle still contributes appreciably to the total X-ray absorption signals.

With the polar component $\left(\theta_{B}\right)$ of the $\mathrm{CO}$ bond vector fixed at $30^{\circ}$, we also tested whether the $I$ versus $\theta$ curve of the second particle is modified by varying the azimuthal component $\left(\phi_{B}\right)$ of the $\mathrm{CO}$ bond vector. As shown in Fig. 7d, varying $\phi_{B}$ only causes a small change to the $I$ versus $\theta$ curve. By examining the individual $I$ versus $\theta$ curves obtained from different parts of the particle, we found that those of the top and side facets are unaffected by varying $\phi_{B}$, presumably due to the three/four-fold rotation symmetry inherent in $\{111\} /\{100\}$ facets. On this basis, we attribute the $\phi_{B}$-dependence of the $I$ versus $\theta$ curve to $\mathrm{CO}$ on the edge and corner sites of the particle where rotation symmetry does not exist.

\subsection{Versus $\beta$ Curve}

\subsubsection{Individual I Versus $\beta$ Curves from Different Parts of the Hypothetical Nanoparticle}

The bond orientation of $\mathrm{CO}$ on a nanoparticle can also be determined by measuring the $\mathrm{C}-\mathrm{O} \pi^{*}$ resonance peak 
(a)

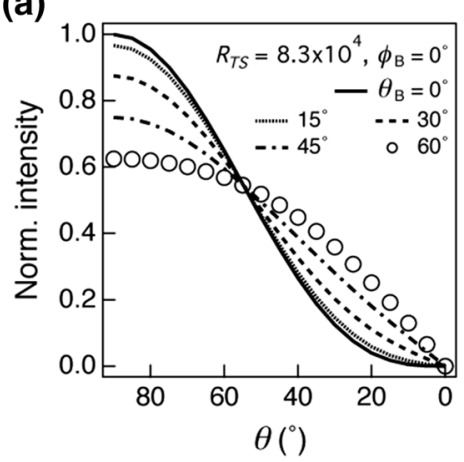

(c)

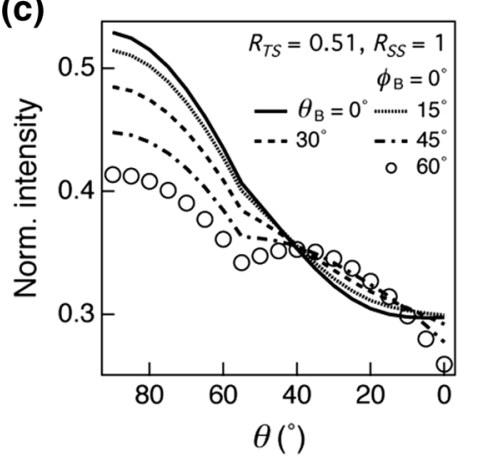

(b)

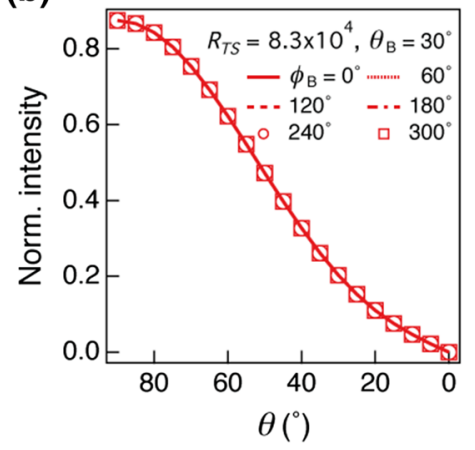

(d)

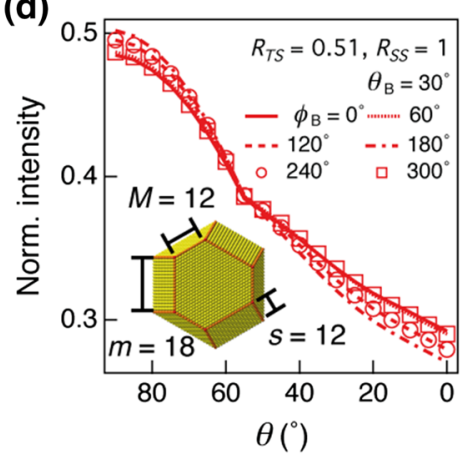

(e)

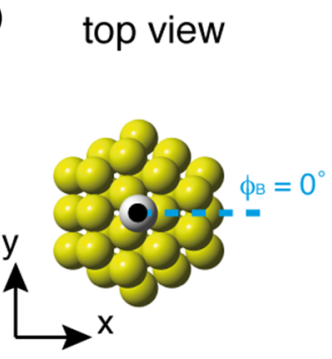

(i) $\mathrm{B}_{\mathrm{co}}=\mathrm{N}$ side view

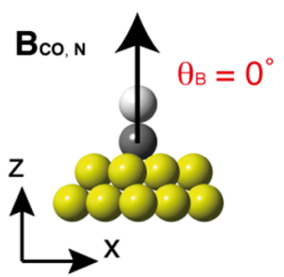

$\theta_{\mathrm{B}}=0^{\circ}, \phi_{\mathrm{B}}=0^{\circ}$

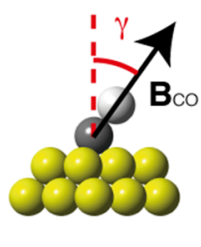

(ii) Rotate $\mathbf{B}_{\mathrm{co}}$ about the $\mathrm{y}$-axis by $\gamma \quad \theta_{\mathrm{B}}=\gamma, \phi_{\mathrm{B}}=0^{\circ}$
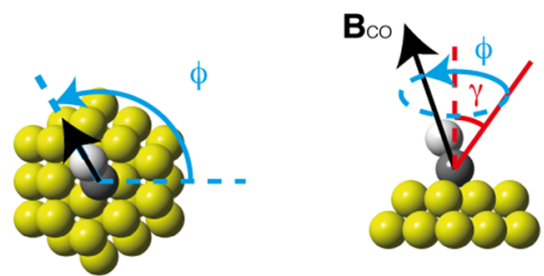

(iii) Rotate $\mathbf{B}_{c o}$ about the $z$-axis by $\phi \quad \theta_{\mathrm{B}}=\gamma, \phi_{\mathrm{B}}=\phi$

Fig. 7 Simulated $I$ versus $\theta$ curves obtained from two different particles on which $\mathrm{CO}$ is assumed to bond with its molecular axis away from the corresponding facet (edge or corner) normal vector, hence $\mathbf{B}_{\mathbf{j}} \neq \mathbf{N}_{\mathbf{j}}$ for all $j$. In $\mathbf{a}, \mathbf{b}$, the particle is defined by $(M, m$, $s)=\left(10^{6}, 3,3\right)$ and $R_{T S}=8.3 \times 10^{4}$, hence mimicking a native (111) surface. In $\mathbf{c}, \mathbf{d}$ the particle is defined as $(M, m, s)=(12,18,12)$ and has $R_{T S}=0.51$ and $\mathrm{R}_{\mathrm{SS}}=1$ and its (111) top facet as well as the $\{111\}$ side facets and $\{100\}$ side facets of equal size. In a, c, CO is set to bond at varying polar angle $\left(\theta_{B, j}\right)$ away from the corresponding

intensity as a function of the direction of the electric field vector $\mathbf{E}$ (hence the polarization angle, $\beta$ ) of the incident photon beam. In these measurements, the sample position and the direction of the incident photon beam are fixed so that the photon doses on different parts of a particle are unchanged as $\beta$ varies.

Figure 8 displays the individual $I$ versus $\beta$ curves simulated from different parts of a particle that has parameters $(M, m, s)=(12,8,4)$. In the simulation, the particle was orientated in the same way shown in Fig. 1 b, i.e. $\mathbf{N}_{\text {particle }}$ $=\left(1,0^{\circ}, 0^{\circ}\right)$. The incident photon beam has a direction of $\mathbf{P}=\left(1,106^{\circ} 180^{\circ}\right)$, corresponding to an incidence angle of $\theta=16^{\circ}$, with the direction of its electric field vector $\mathbf{E}$ governed by the polarization angle $\beta$. Note that $\beta=90^{\circ}$ $\left(0^{\circ}\right)$ corresponds to $s-(p-)$ polarized light. To simplify the simulation, we first assumed that $\mathrm{CO}$ bonds with its molecular axis parallel to the corresponding facet (edge or corner) normal, therefore $\mathbf{B}_{\mathbf{j}}=\mathbf{N}_{\mathbf{j}}$ for all $j$. Due to the facet (edge or corner) normal vector, while in $(\mathbf{b}, \mathbf{d})$, with the polar angle fixed at $\theta_{B, j}=30^{\circ}, \mathrm{CO}$ is set to bond at varying azimuthal angle $\left(\phi_{B, j}\right)$ with respect to the corresponding facet (edge or corner) normal vector. The particles are oriented as in Fig. 1b. The direction of the incident photon beam is fixed at $\mathbf{P}=\left(1,180^{\circ}, 180^{\circ}\right)$. The incident light is set to be $p$-polarized, hence $\beta=0^{\circ}$. e Schematic depiction of angles $\theta_{B}$ and $\phi_{B}$ that define the direction of the $\mathrm{CO}$ bond vector $\left(\mathbf{B}_{\mathbf{C O}, \mathbf{j}}\right)$ relative to the normal vector of the correspond facet $j\left(\mathbf{N}_{\mathbf{j}}\right)$

different orientations of $\mathrm{CO}$ relative to the incident photon beam at different parts of the particle, the individual $I$ versus $\beta$ curves are markedly different from each other. Summing all the individual curves in Fig. $8 \mathrm{~b}-\mathrm{h}$ gives the total $I$ versus $\beta$ curve of the particle, which, as mentioned in Part II, is then normalized to the number of surface atoms on the particle. The normalized $I$ versus $\beta$ curve is shown in Fig. 8a. We will discuss how the overall $I$ versus $\beta$ curve of a particle is modified by factors such as the particle geometry and orientation as well as the $\mathrm{CO}$ bond vector.

\subsubsection{Nanoparticle Aspect Ratio and Orientation}

Figure 9a displays the $I$ versus $\beta$ curves obtained from particles having the same $m$ and $s$ value $(m=s=3)$ but different $M$ values. For a particle having $M=10^{6}$ hence $R_{T S}=8.3 \times 10^{4}$, the corresponding $I$ versus $\beta$ curve 


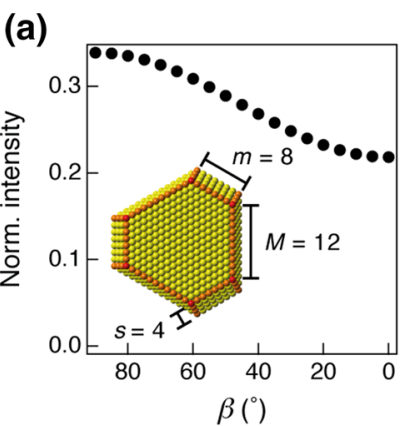

(e)

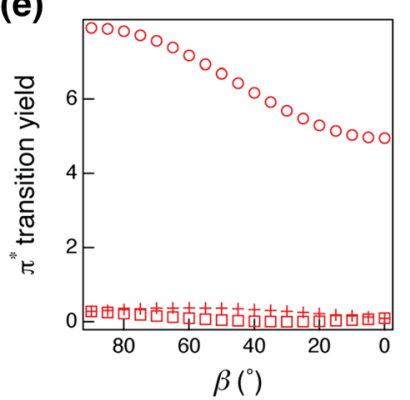

Edges between (111) top facet \& $\{111\}$ side facets

$\circ(1 \overline{1} 1) \quad \square(\overline{1} 11)+(11 \overline{1})$ (b)

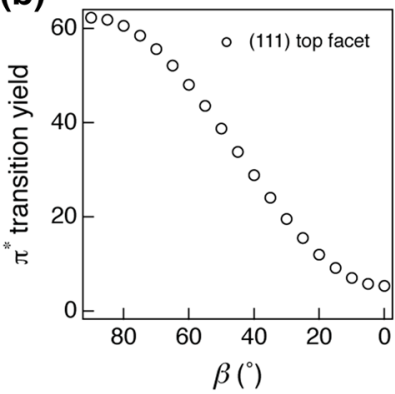

(f)

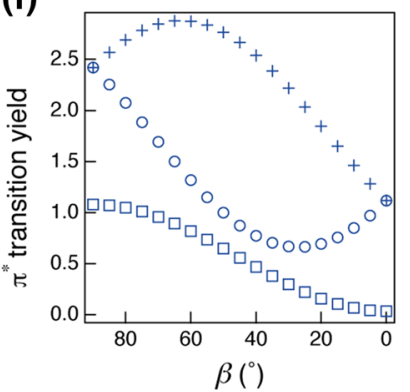

Edges between (111) top facet \& $\{100\}$ side facets

$\circ(010) \square(001)+(100)$ (c)

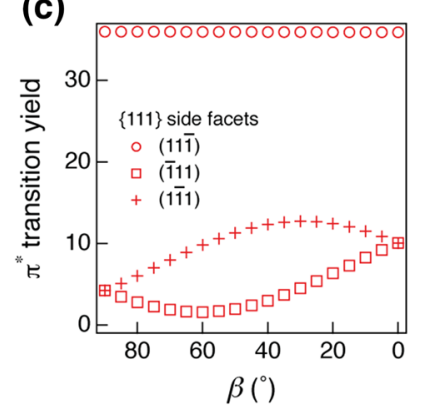

(g)

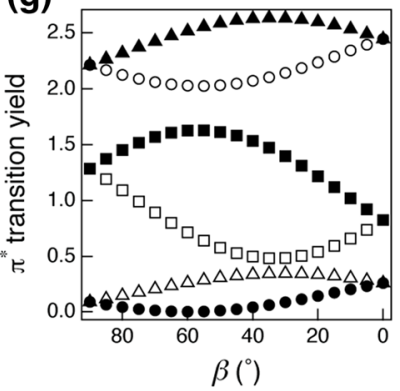

Edges between $\{111\} \&\{100\}$ side facets

$\begin{array}{lll}0 & (11 \overline{1}) \&(010) & \bullet(\overline{1} 11) \&(010) \\ \square & (\overline{1} 11) \&(001) & \square(1 \overline{1} 1) \&(001)\end{array}$

ㅁ $(\overline{1} 11) \&(001)$

- $\quad(1 \overline{1} 1) \&(001)$ (d)

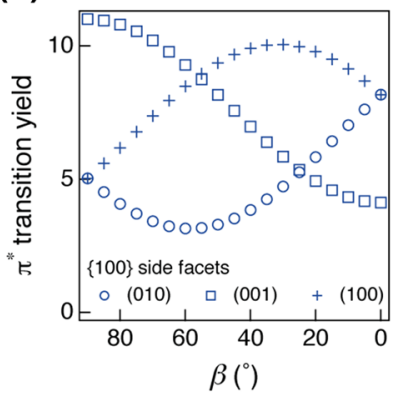

(h)

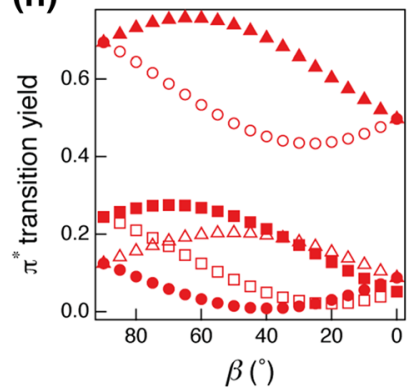

Corners between (111) top facet, $\{111\}$

\& $\{100\}$ side facets

- $(11 \overline{1}) \&(010)$

(111) \& (001)

Fig. 8 Simulated $\pi^{*}$ resonance intensity versus polarization angle $(I$ versus $\beta$ ) curves obtained from different parts of a CO-covered nanoparticle constructed using parameters $(M, m, s)=(12,8,4)$. a The total curve that is a summation over curves from different parts of the particle (illustrated in the inset) including: b (111) top facet, $\mathbf{c}\{111\}$ side facets, $\mathbf{d}\{100\}$ side facets, e edges between the (111) top and $\{111\}$ side facets, $\mathbf{f}$ edges between the (111) top and $\{100\}$ side facets, $\mathbf{g}$ edges between $\{111\}$ and $\{100\}$ side facets, and $\mathbf{h}$ corner sites. After summation, the total curve is normalized

decreases monotonically with decreasing $\beta$. This curve resembles that of $\mathrm{CO}$ on a $\operatorname{Pd}(111)$ single crystal surface. As $M$ decreases to small values, the $I$ versus $\beta$ curve varies in several ways: in addition to an upward shift in intensity at $\beta=90^{\circ}$ (s-polarized), the curve also drops at a descending rate, resulting in a higher intensity at $\beta=0^{\circ}$ ( $p$-polarized). This overall upward shift is due to the increasing signal contributions of $\mathrm{CO}$ on the side facets, edges and corner sites of the particle, which due to their different orientations relative to the incident photon beam, interacts with the incident photon beam differently compared with $\mathrm{CO}$ on the (111) top facet.

We also examined how the $I$ versus $\beta$ curve of a nanoparticle is affected by the crystal rotation. Two particles were used: one defined as $(M, m, s)=\left(10^{6}, 3,3\right)$ resembling a $\operatorname{Pd}(111)$ single crystal surface while the other has parameters $(M, m, s)=(12,18,12)$ and has almost equal numbers of atoms $(\sim 30 \%)$ located on the (111) top facet, $\{111\}$ side facets, and $\{100\}$ side facets. As shown in Fig. $9 \mathrm{~b}$, the $I$ versus $\beta$ curve of the first particle does not change with crystal orientation. This is expected because

to the number of surface atoms of the particle. In the simulation, the particle is oriented in the same manner as shown in Fig. 1b,

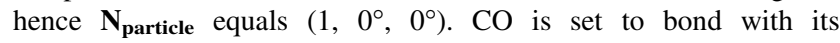
molecular axis parallel to the facet normal, hence $\mathbf{B}_{\mathbf{j}}=\mathbf{N}_{\mathbf{j}}$ for all $j$. The polar $\left(\theta_{P}\right)$ and azimuthal angles $\left(\phi_{P}\right)$ of the incident photon beam are fixed at $106^{\circ}$ and $180^{\circ}$ respectively. This corresponds to a grazing incident angle of $\theta=16^{\circ}$. Note that $\beta=90^{\circ}\left(0^{\circ}\right)$ means that the incident photon beam is completely $s-(p-)$ polarized

when $\mathrm{CO}$ is bonded upright on a semi-infinite surface, rotating the surface about its normal causes no change to the bond orientation of $\mathrm{CO}$ and hence the $\pi^{*}$ resonance intensity signal is not affected.

For the second particle, the $I$ versus $\beta$ curve in Fig. $9 \mathrm{c}$ is obviously affected by the azimuthal rotation. This is because when the particle rotates, the orientations of different parts of the particle as well as the corresponding $\mathrm{CO}$ vectors rotate correspondingly. This affects both the photon doses and the interactions between the incident photon beam and $\mathrm{CO}$ on different facets (edges and corners) of the particle, and hence the overall $I$ versus $\beta$ curve. The $I$ versus $\beta$ curves obtained at $\theta_{\text {particle }}=0^{\circ}$ and $\theta_{\text {particle }}=120^{\circ}$ are identical due to the threefold rotation symmetry of the particle.

\subsubsection{Varying CO Bond-Vector}

As we saw in the previous section, varying the particle orientation affects the $I$ versus $\beta$ curve partly because the $\mathrm{CO}$ orientation changes. Therefore, directly varying the $\mathrm{CO}$ 
(a)

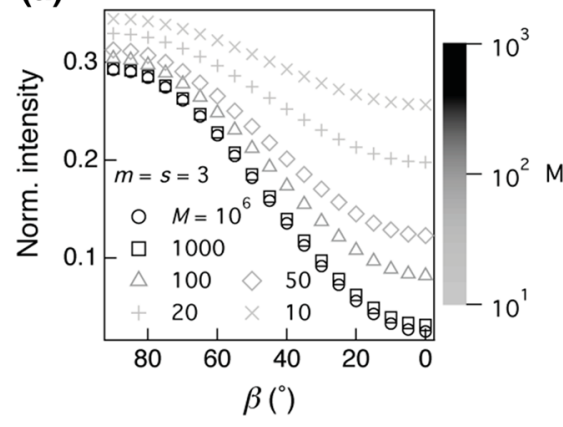

(b)

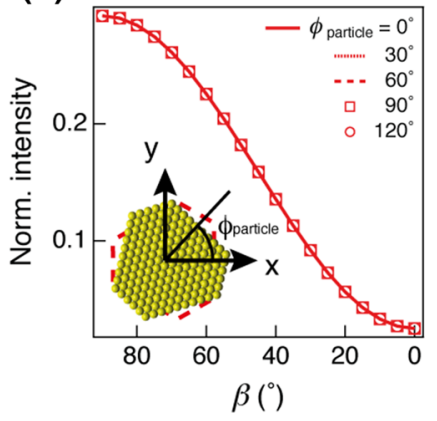

(c)

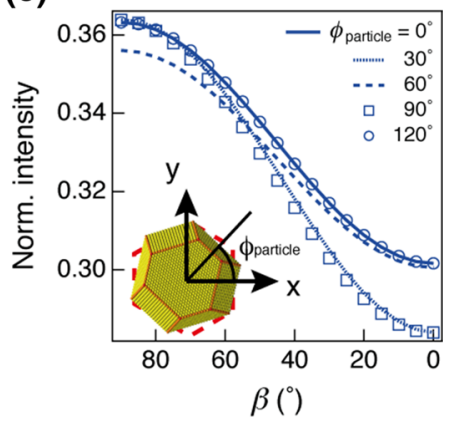

Fig. 9 a Simulated $I$ versus $\beta$ curves obtained from the CO-covered particles that are constructed with the same values of $m=3$ and $s=3$ but different $M$ values: $10^{6}$ (circle), $10^{3}$ (square), 100 (triangle), 50 (diamond), 20 (plus) and 10 (cross). All particles are oriented in the same manner as shown in Fig. 1 b, hence $\mathbf{N}_{\text {particle }}$ $=\left(1,0^{\circ}, 0^{\circ}\right)$. $\mathbf{b}$ Simulated $I$ versus $\beta$ curves obtained from the COcovered particle that is constructed using parameters $(M, m$, $s)=\left(10^{6}, 3,3\right)$ and is rotated by different angles about the particle normal vector. This particle has a large value of $R_{T S}=8.3 \times 10^{4}$ and therefore mimics a native (111) surface. c Simulated $I$ versus $\beta$ curves obtained from a particle that is constructed using parameters $(M, m$,

bond orientation should also affect the $I$ versus $\beta$ curve of the particle and we shall see how the curve changes here. The same two nanoparticles are used as in Sect. 3.2.2.

The first particle resembles a single crystal $\operatorname{Pd}(111)$ sample. As shown in Fig. 10a, varying the polar angle $\left(\theta_{B}\right)$ of the $\mathrm{CO}$ bond vector $\left(\mathbf{B}_{\mathbf{C O}, \mathbf{j}}\right.$ for all $j$ ) with respect to the normal vector of each of the facets, edges and corners $\left(\mathbf{N}_{\mathbf{j}}\right.$ for all $j$ ) with the azimuthal component fixed at $\phi_{B}=0^{\circ}$ alters the $I$ versus $\beta$ curve in several ways. Apart from having a decreasing intensity at $\beta=90^{\circ}$ (s-polarized), the curve drops monotonically with a decreasing rate and hence reaches an increased intensity value at $\beta=0^{\circ}$ ( $p$ polarized). This character holds up to $\theta_{B}=54.74^{\circ}$, after which the $I$ versus $\beta$ curve inverts its dependence on $\beta$. Note that at $\theta_{B}=54.74^{\circ}$, the $\mathrm{CO} \pi^{*}$ resonance intensity is almost independent of the polarization angle $\beta$ of the incident photon beam. This corresponds to the 'magic' polar bond angle of $\mathrm{CO}$ on the native (111) surface [11]; at this angle, the $\mathrm{CO}$ molecules bonding at three equivalent azimuthal directions, relative to the surface normal, interact with the incident photon beam to give an overall $\pi^{*}$ resonance intensity whose dependence on the direction of the electric field vector $\mathbf{E}$ is cancelled out.

With the polar component of the $\mathrm{CO}$ bond vector fixed at $\theta_{B}=30^{\circ}$, we also tested how the $I$ versus $\beta$ curve of the first particle is affected by varying the azimuthal component $\left(\phi_{B}\right)$ of the $\mathrm{CO}$ bond vector. As evidenced in Fig. 10b, the $I$ versus $\beta$ curve of this crystal does not change with $\phi_{B}$. This is in good agreement with $\mathrm{CO}$ on a surface of a single crystal, where the dependence of the $\pi^{*}$ resonance signal on the azimuthal dependence is removed by the threefold rotation symmetry of the surface. $s)=(12,18,12)$ and is rotated by different angles about the particle normal vector. The particle has $R_{T S}=0.51$ and $R_{S S}=1$. Before rotation (i.e. at $\phi_{\text {particle }}=0^{\circ}$ ), the particles are oriented as in Fig. 1b. $\mathrm{CO}$ is taken to bond with its molecular axis parallel to the corresponding facet (edge or corner) normal, hence $\mathbf{B}_{\mathbf{j}}=\mathbf{N}_{\mathbf{j}}$ for all $j$. The polar $\left(\theta_{P}\right)$ and azimuthal angles $\left(\phi_{P}\right)$ of the incident photon beam are fixed at $106^{\circ}$ and $180^{\circ}$ respectively. This corresponds to a grazing incident angle of $\theta=16^{\circ}$. Note that $\beta=90^{\circ}\left(0^{\circ}\right)$ means that the incident photon beam is completely $s$ - $(p-)$ polarized. Each particle is illustrated in the inset, with its orientation before azimuthal rotation marked with red dashed lines

The second particle has almost equal amount of atoms in the (111) top facet, $\{111\}$ side facets and $\{100\}$ side facets. The $\theta_{B}$-dependence of its $I$ versus $\beta$ curve are shown in Fig. 10c. Before normalization, the intensity ranges for Fig. 10a, c are 0.267 and 0.062 , respectively. This indicates that the $\pi^{*}$ resonance of the second crystal is, in general, less dependent upon $\beta$. In addition, its dependence on the polar component of the $\mathrm{CO}$ bond-vector $\left(\theta_{B}\right)$ is also different: while the $I$ versus $\beta$ curve of the second particle changes in shape with increasing $\theta_{B}$ in a similar fashion to that of the first particle, it shifts upward with increasing $\theta_{B}$ much more slowly. Moreover, although on this particle there also exists a $\theta_{B}$ value $\left(=50.8^{\circ}\right)$ at which the $\pi^{*}$ resonance intensity does not vary with $\beta$, we found no $\beta$ value at which the $\pi^{*}$ resonance intensity is invariant with $\theta_{B}$. On the other hand, Fig. 10a shows that for the first particle at $\beta=53^{\circ}$ the $\pi^{*}$ resonance intensity is the same for all values of $\theta_{B}$. These differences originate from the different shapes of the particles. While on the first particle only CO on the (111) top facet contributes to the X-ray absorption signal, on the second particle $\mathrm{CO}$ on the side facets also contributes. Also, due to their different orientations relative to the incident photon beam, the $\pi^{*}$ resonance signals arising from various parts of the particle are also different. All these factors explain why the $I$ versus $\beta$ curves of the two particles, as well as their dependence on $\theta_{B}$, are so distinct from each other.

With the polar component fixed at $\theta_{B}=30^{\circ}$, we also tested how the $I$ versus $\beta$ curve of the second particle varies with the azimuthal orientation of $\mathrm{CO}\left(\phi_{B}\right)$. As shown in Fig. 10d, its $I$ versus $\beta$ curve alters in shape with $\phi_{B}$. By examining the individual $I$ versus $\beta$ curves, we find that 


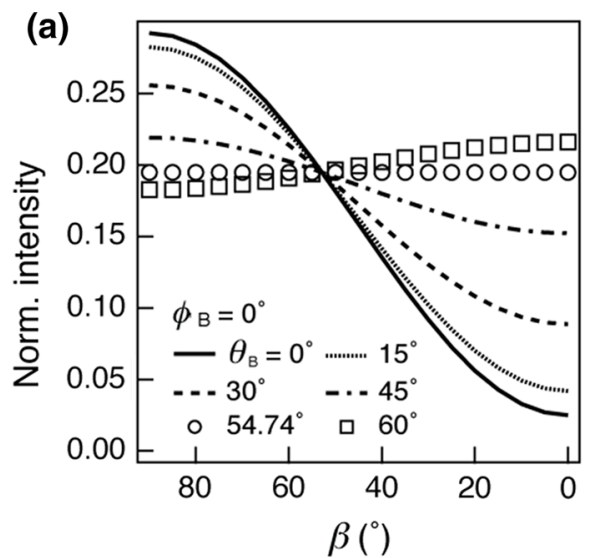

(c)

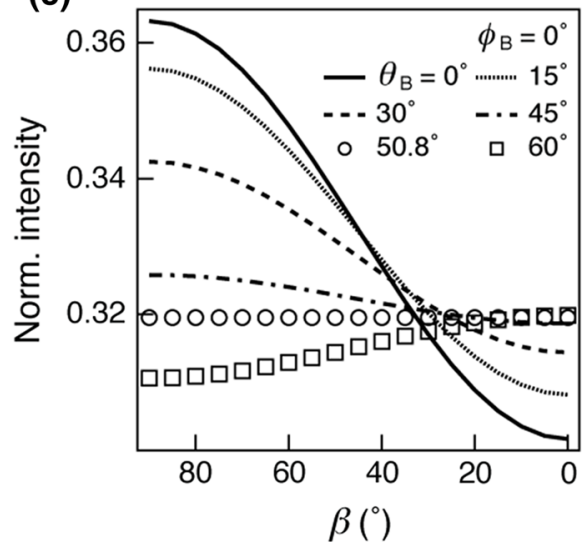

Fig. 10 Simulated $I$ versus $\beta$ curves obtained from two different particles on which $\mathrm{CO}$ is assumed to bond with its molecular axis away from the corresponding facet (edge or corner) normal vector, hence $\mathbf{B}_{\mathbf{j}} \neq \mathbf{N}_{\mathbf{j}}$ for all $j$. In (a-b), the particle that is defined as $(M, m$, $s)=\left(10^{6}, 3,3\right)$ with $R_{T S}=8.3 \times 10^{4}$, hence mimicking a native (111) surface, while in $\mathbf{c}, \mathbf{d}$ the particle is defined by $(M, m, s)=(12$, $18,12)$ with $R_{T S}=0.51$ and $R_{S S}=1$, so that its $(111)$ top facet as well as the $\{111\}$ side facets and $\{100\}$ side facets are of equal size. The shape of the second particle is shown in the inset of $\mathbf{d}$. In a, c, CO is set to bond at varying polar angle $\left(\theta_{B, j}\right)$ away from the corresponding facet (edge or corner) normal vector, while in $\mathbf{b}, \mathbf{d}$,

those of the top and side facets are unaffected by changing $\phi_{B}$. Thus, we attribute this $\phi_{B}$-dependence to $\mathrm{CO}$ on the edge and corner sites.

\subsection{Comparison Between Simulation and Experiment}

In this section, we apply our model to a set of carbon K-edge NEXAFS data that were obtained on a Pd nanoparticle covered with $\mathrm{CO}$ [28]. The experiments were performed using the SPELEEM end-station of Beamline I06, Diamond Light Source (UK). We measured the $\pi^{*}$ resonance intensity $(I)$ originating from $\mathrm{CO}$ adsorbed on the nanoparticle, as a function of the polarization angle $(\beta)$ of the incident light with the angle of incidence fixed at
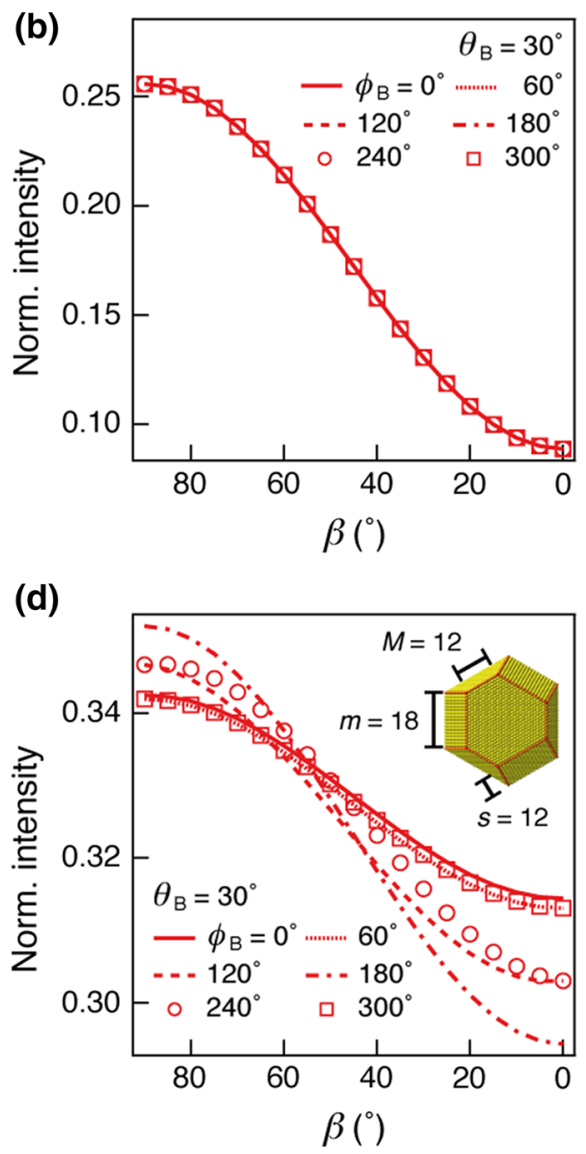

with the polar angle fixed at $\theta_{B, j}=30^{\circ}, \mathrm{CO}$ is set to bond at varying azimuthal angle $\left(\phi_{B, j}\right)$ with respect to the corresponding facet (edge or corner) normal vector. In the simulation, the particles are oriented as in Fig. 1b. The polar $\left(\theta_{P}\right)$ and azimuthal angles $\left(\phi_{P}\right)$ of the incident photon beam are fixed at $106^{\circ}$ and $180^{\circ}$ respectively. This corresponds to a grazing incident angle of $\theta=16^{\circ} . \beta=90^{\circ}\left(0^{\circ}\right)$, indicating that the incident photon beam is $s$ - $(p$-) polarized. A schematic depiction of angles $\theta_{B}$ and $\phi_{B}$ of the $\mathrm{CO}$ bond vector $\left(\mathbf{B}_{\mathbf{C o}}\right)$ relative to the normal vector of the corresponding facet $j\left(\mathbf{N}_{\mathbf{j}}\right)$ can be found in Fig. 7e

$16^{\circ}$. When fitted with appropriate equations adapted from Ref. [11] with the assumption that only CO on the top facet contributes to the absorption signal, the data reveal a $\mathrm{CO}$ bond angle $21.4^{\circ} \pm 7.0^{\circ}$ away from the surface normal. This is in poor agreement with a previous NEXAFS study by Knight et al. [27] which shows that CO bonds vertically on a $\operatorname{Pd}(111)$ surface. This therefore suggests that there are significant contributions from $\mathrm{CO}$ on other parts of the nanoparticle, which we will evaluate using the methods described in the previous sections.

The experimental data and the accompanying numerical fit sourced from Fig. 3b of Ref. [28] are re-plotted in Fig. 11a. The experimental data (squares) and numerical fit (solid line) together serve as the 'fitting curve' for the simulations which follow. In order to take the experimental 
limitations into account, we normalize all the $I$ versus $\beta$ curves presented here so that they all start at a unity intensity value at $\beta=90^{\circ}$ ( $s$-polarised). Regarding the nanoparticle shape used in the simulation, previous STM experiments have shown that $\mathrm{Pd}$ nanoparticles that are grown on $\mathrm{TiO}_{2}(110)$ using a method similar to that employed in the NEXAFS measurements in Ref. [28] are pseudo-triangular in shape. They are therefore characterized by a $\{111\}$ top facet with $\{111\}$ and $\{100\}$ side facets [29]. We assume that all the nanoparticles used for the simulations here are characterized by the same shape and are oriented in the same manner as shown in Fig. 1b. Assuming that $\mathrm{CO}$ bonds vertically on each of the different parts of the nanoparticle (hence $\mathbf{B}_{\mathbf{j}}=\mathbf{N}_{\mathbf{j}}$ for all $j$ ), we searched for nanoparticles with different $(M, m, s)$ values

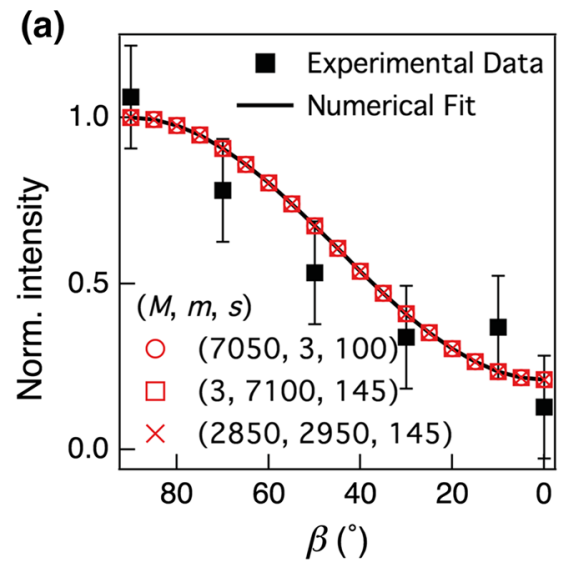

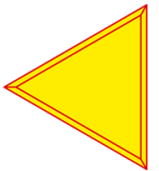

$(7050,3,100)$

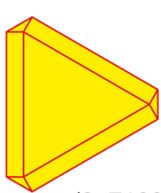

$(3,7100,145)$

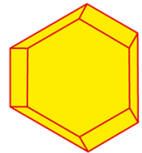

$(2850,2950,145)$ that have an average diameter of $\sim 1.5 \mu \mathrm{m}$ and whose $I$ versus $\beta$ curves match the 'fitting' curve. We found several nanoparticles whose $I$ versus $\beta$ curves overlap perfectly with the 'fitting' curve (solid line), three of which are plotted as (circles, squares and crosses). These nanoparticles are defined by $(M, m, s)=(7050,3,100),(3$, $7100,145)$ and $(2850,2950,145)$ and have an average $R_{T S}$ value of $10.0 \pm 1.8$, an average height $(h)$ of $2.91 \pm 5.8 \mathrm{~nm}$ and hence an average aspect ratio $(d / h)$ of $51.8 \pm 11.2$. Although these nanoparticles have rather different shapes, they share one common feature: all of them are very thin particles. This means that $\mathrm{CO}$ on the top (111) facet provides the major contribution to the X-ray absorption signal.

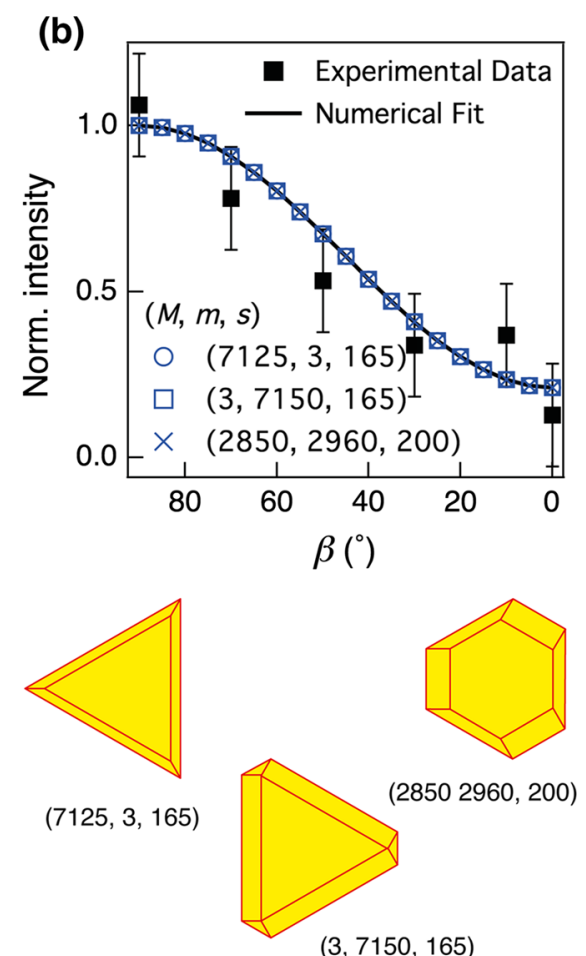

$(7125,3,165)$

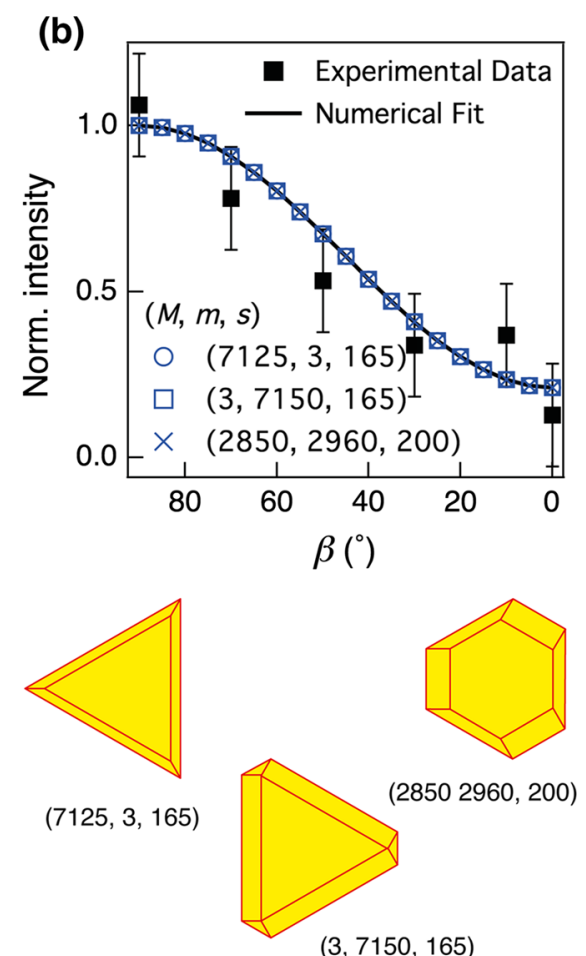

$(3,7150,165)$
Fig. 11 a A comparison between the experimental data and the simulated $I$ versus $\beta$ curves obtained from nanoparticles of different shapes. Black solid squares are the experimental data that were obtained from $\mathrm{C}$ K-edge NEXAFS measurements $(\mathrm{h} v=285-295 \mathrm{eV})$ on an isolated Pd nanoparticle (average diameter $=1.5 \mu \mathrm{m})$ supported on $\mathrm{TiO}_{2}(110)$, recorded following a $\mathrm{CO}$ exposure of 100 Langmuirs $\left(1 \mathrm{~L}=1.33 \times 10^{-6} \mathrm{mbar} \mathrm{s}\right)$ at $300 \mathrm{~K}$; the black solid line is the numerical fit to the experimental data using appropriate equations from Ref. [11]. With the assumption of CO bonding to only the top facet, this reveals a polar bond angle $\left(\theta_{B}\right)$ of $21.4^{\circ} \pm 7.0^{\circ}$ [28]. Other experimental details regarding the acquisition and analysis of the NEXAFS data can be found in Ref. [28]. Red markers are the simulated $I$ versus $\beta$ curves that are obtained from nanoparticles constructed using different sets of $(M, m, s)$ parameters that give an average particle diameter of $\sim 1.5 \mu \mathrm{m}(d)$. The shapes of the nanoparticles used in the simulations are shown at the bottom part of the figure. These curves match perfectly with the numerical fit (black solid line). On the nanoparticles, $\mathrm{CO}$ is taken to bond with its molecular axis parallel to the facet normal, hence $\mathbf{N}_{\mathbf{j}}=\mathbf{B}_{\mathbf{j}}$ for all $j$. The nanoparticles whose $I$ versus $\beta$ curves match perfectly with the numerical fit have an average $R_{T S}$ value of $10.0 \pm 1.8$, an average height $(h)$ of $29.1 \pm 5.8 \mathrm{~nm}$, giving an average aspect ratio $(d / h)$ of $51.8 \pm 11.2$. b As a, with signal contribution of $\mathrm{CO}$ on the facets (edges or corners) not in line-of-sight set to zero to simulate full attenuation of X-ray within the particle. As a result, the nanoparticles whose $I$ versus $\beta$ curves (blue markers) match perfectly with the numerical fit (black solid line) have different $(M, m, s)$ values. An average $R_{T S}$ value of $7.2 \pm 0.02$ and an average height $(h)$ of $39.6 \pm 4.6 \mathrm{~nm}$ are found with this adjustment, giving a lower average aspect ratio of $37.7 \pm 4.1$. In the simulation, the particles are oriented as in Fig. 1b. The polar $\left(\theta_{P}\right)$ and azimuthal angles $\left(\phi_{P}\right)$ of the incident photon beam are fixed at $106^{\circ}$ and $180^{\circ}$, respectively. This corresponds to a grazing incident angle of $\theta=16^{\circ}$ 
The average diameter of the (CO-covered) nanoparticle employed for the CO $\pi^{*}$ NEXAFS measurements is $\sim 1.5 \mu \mathrm{m}$ [28], ten times larger than the attenuation length of the X-rays $(0.151 \mu \mathrm{m})$. As such, the incident photon beam cannot transmit through the nanoparticle, and as a result, $\mathrm{CO}$ molecules bound on the side facets not in lineof-sight to the photon beam do not contribute to the X-ray absorption signal at all. To account for this, we excluded contributions from $\mathrm{CO}$ on the side facets (edges and corners) that are not in line-of-sight to the photon beam, and searched again for nanoparticles whose $I$ versus $\beta$ curves match perfectly with the fitting curve. As displayed in Fig. $11 \mathrm{~b}$, three particles (amongst others) whose $I$ versus $\beta$ curves (circles, squares and crosses respectively) overlap perfectly with the fitting curve are plotted. The nanoparticles are defined as $(M, m, s)=(7125,3,165),(3,7150$, $165)$ and $(2850,2960,200)$ and have a reduced averaged $R_{T S}$ value of $7.2 \pm 0.02$. We therefore deduce that the nanoparticle observed in PEEM should have a height of $39.6 \pm 4.6 \mathrm{~nm}$, giving an aspect ratio $(d / h)$ of $37.7 \pm 4.1$. This suggests that the Pd nanoparticle is a thin island.

\section{Summary}

We have developed an analytical method to simulate the carbon K-edge NEXAFS measurements on a $\mathrm{TiO}_{2}(110)$ supported Pd nanoparticle covered with CO. The method can be adapted to simulate other adsorbate-nanoparticle systems. In the case of Pd, the nanoparticle is defined with a (111) top facet as well as three equally sized $\{111\}$ and $\{100\}$ side facets. Two experimental geometries have been considered. In the first geometry, by fixing the polarization angle $\beta$, a curve of the $\mathrm{CO} \pi^{*}$ resonance intensity $(I)$ as a function of the incident angle $\theta$ was generated. This geometry corresponds to the standard geometry as used in connection with a conventional synchrotron radiation source. In the second geometry that models a typical XPEEM experiment with a double-undulator, to rotate the electric field vector $\mathbf{E}$ of the X-rays we fixed the angle of incidence $\theta$ and calculated $I$ versus $\beta$ curves. In both geometries, we examined the dependence of these curves on the particle size and shape, the azimuthal orientation of the particle with respect to the incident photon beam, and the bonding orientation of CO. Finally, we applied our model to analyze a set of carbon K-edge NEXAFS data obtained from a single, CO-covered Pd nanoparticle that was supported on $\mathrm{TiO}_{2}(110)$. Our simulation predicts the nanoparticle is thin, with a high aspect ratio of $37.7 \pm 4.1$.

Acknowledgments This work was supported by EPSRC (UK), EU COST Action CM1104, an ERC Advanced Grant (ENERGY SURF) and the Royal Society.
Open Access This article is distributed under the terms of the Creative Commons Attribution 4.0 International License (http://crea tivecommons.org/licenses/by/4.0/), which permits unrestricted use, distribution, and reproduction in any medium, provided you give appropriate credit to the original author(s) and the source, provide a link to the Creative Commons license, and indicate if changes were made.

\section{References}

1. Benedetti S, Myrach P, di Bona A et al (2011) Growth and morphology of metal particles on $\mathrm{MgO} / \mathrm{Mo}(001)$ : a comparative STM and diffraction study. Phys Rev B 83:125423

2. Højrup-Hansen K, Ferrero S, Henry CR (2004) Nucleation and growth kinetics of gold nanoparticles on $\mathrm{MgO}(100)$ studied by UHV-AFM. Appl Surf Sci 226:167-172

3. Humphrey DS, Cabailh G, Pang CL et al (2009) Self-assembled metallic nanowires on a dielectric support: $\mathrm{Pd}$ on rutile $\mathrm{TiO}_{2}(110)$. Nano Lett 9:155-159

4. Liu Q, Bauer JC, Schaak RE, Lunsford JH (2008) Supported palladium nanoparticles: an efficient catalyst for the direct formation of $\mathrm{H}_{2} \mathrm{O}_{2}$ from $\mathrm{H}_{2}$ and $\mathrm{O}_{2}$. Angew Chem 120:6317-6320

5. Renaud G (2003) Real-time monitoring of growing nanoparticles. Science 300:1416-1419

6. Revenant C, Leroy F, Lazzari R et al (2004) Quantitative analysis of grazing incidence small-angle $\mathrm{x}$-ray scattering: $\mathrm{Pd} / \mathrm{MgO}(001)$ growth. Phys Rev B 69:035411

7. Højrup-Hansen K, Šljivančanin Ž, Lægsgaard E et al (2002) Adsorption of $\mathrm{O}_{2}$ and $\mathrm{NO}$ on $\mathrm{Pd}$ nanocrystals supported on $\mathrm{Al}_{2} \mathrm{O}_{3} / \mathrm{NiAl}(110)$ : overlayer and edge structures. Surf Sci 505:25-38

8. Viñes F, Desikusumastuti A, Staudt T et al (2008) A combined density-functional and IRAS study on the interaction of NO with Pd nanoparticles: identifying new adsorption sites with novel properties. J Phys Chem C 112:16539-16549

9. Peter M, Flores Camacho JM, Adamovski S et al (2013) Trends in the binding strength of surface species on nanoparticles: how does the adsorption energy scale with the particle size? Angew Chem Int Ed 52:5175-5179

10. Stöhr J (1992) NEXAFS spectroscopy. Springer, Berlin

11. Stöhr J, Outka DA (1987) Determination of molecular orientations on surfaces from the angular dependence of near-edge x-ray-absorption fine-structure spectra. Phys Rev B 36:7891

12. Davis R, Lindsay R, Purcell KG et al (1991) A NEXAFS study of the orientation of $\mathrm{CO}$ on $\mathrm{Cu}(110)$. J Phys: Condens Mat 3:S297S302

13. Cappus D, Klinkmann J, Kuhlenbeck H, Freund HJ (1995) CO on $\mathrm{NiO}(100)$ : orientation and bonding. Surf Sci 325:L421-L427

14. Beccari M, Kanjilal A, Betti MG et al (2009) Characterization of benzenethiolate self-assembled monolayer on $\mathrm{Cu}(100)$ by XPS and NEXAFS. J Electron Spectrosc Relat Phenom 172:64-68

15. Furukawa M, Yamada T, Katano S et al (2007) Geometrical characterization of adenine and guanine on $\mathrm{Cu}(110)$ by NEXAFS, XPS, and DFT calculation. Surf Sci 601:5433-5440

16. Syres K, Thomas A, Bondino F et al (2010) Dopamine adsorption on anatase $\mathrm{TiO}_{2}(101)$ : a photoemission and NEXAFS spectroscopy study. Langmuir 26:14548-14555

17. Imanishi A, Suzuki H, Ohashi N et al (2009) Pretreatment dependence of adsorption properties of merocyanine dye at rutile (110) and (100) $\mathrm{TiO}_{2}$ surfaces studied by C K-Edge NEXAFS. J Phys Chem C 113:17254-17261

18. Lindsay R, Guitiérrez-Sosa A, Thornton G et al (1999) NEXAFS study of $\mathrm{CO}$ adsorption on $\mathrm{ZnO}(000 \overline{1})-\mathrm{O}$ and $\mathrm{ZnO}(000 \overline{1})-\mathrm{O} / \mathrm{Cu}$. Surf Sci 439:131-138 
19. Cabailh G, McGovern IT, Vearey-Roberts A et al (2005) Growth of metal-phthalocyanine on $\operatorname{GaAs}(001)$ : an NEXAFS study. OPTO-Ireland SPIE Proc 5826:37-43

20. Lee H-K, Park J, Kim I et al (2012) Selective reactions and adsorption structure of pyrazine on $\mathrm{Si}(100)$ : HRPES and NEXAFS Study. J Phys Chem C 116:722-725

21. Yim CM, Pang CL, Humphrey DS et al (2013) CO and O overlayers on Pd nanocrystals supported on $\mathrm{TiO}_{2}(110)$. Faraday Discuss 162:191-200

22. Surnev S, Sock M, Ramsey MG et al (2000) CO adsorption on $\operatorname{Pd}(111)$ : a high-resolution core level photoemission and electron energy loss spectroscopy study. Surf Sci 470:171-185

23. Behm RJ, Christmann K, Ertl G, Van Hove MA (1980) Adsorption of CO on Pd(100). J Chem Phys 73:2984

24. Rosenberg HM (1988) The solid state, 3rd edn. Oxford Science Publications, Oxford
25. Henke BL, Gullikson EM, Davis JC (1993) X-Ray interactions: photoabsorption, scattering, transmission, and reflection at $\mathrm{E}=$ 50-30,000 eV, Z = 1-92. At Data Nucl Data Tables 54:181-342

26. Dhesi SS, Cavill SA, Potenza A et al (2010) The nanoscience beamline (I06) at diamond light source. AIP Conf Proc 1234: 311-314

27. Knight MJ, Allegretti F, Kröger EA et al (2008) A structural study of a $\mathrm{C}_{3} \mathrm{H}_{3}$ species coadsorbed with $\mathrm{CO}$ on $\mathrm{Pd}(111)$. Surf Sci 602:2743-2751

28. Yim CM, Pang CL, Hermoso DR et al (2015) Influence of support morphology on the bonding of molecules to nanoparticles. Proc Natl Acad Sci USA 112:7903-7908

29. Wulff G (1901) On the question of speed of growth and dissolution of crystal surfaces. Z Kristallogr 34:449-530 